\title{
THE TRANSPORT OF IMAGES METHOD: COMPUTING ALL ZEROS OF HARMONIC MAPPINGS BY CONTINUATION
}

\author{
Olivier Sète* Jan Zur*
}

May 15, 2021

\begin{abstract}
We present a continuation method to compute all zeros of a harmonic mapping $f$ in the complex plane. Our method works without any prior knowledge of the number of zeros or their approximate location. We start by computing all solution of $f(z)=\eta$ with $|\eta|$ sufficiently large and then track all solutions as $\eta$ tends to 0 to finally obtain all zeros of $f$. Using theoretical results on harmonic mappings we analyze where and how the number of solutions of $f(z)=\eta$ changes and incorporate this into the method. We prove that our method is guaranteed to compute all zeros, as long as none of them is singular. In our numerical example the method always terminates with the correct number of zeros, is very fast compared to general purpose root finders and is highly accurate in terms of the residual. An easy-to-use MATLAB implementation is freely available online.
\end{abstract}

Keywords: harmonic mappings, continuation method, root finding, zeros, Newton's method, critical curves and caustics.

Mathematics subject classification (2020): $65 \mathrm{H} 20 ; 31 \mathrm{~A} 05 ; 30 \mathrm{C} 55$.

\section{Introduction}

We study the zeros of harmonic mappings, i.e., $f: \Omega \rightarrow \mathbb{C}$ with $\Delta f=0$ on an open subset $\Omega$ of $\mathbb{C}$. These functions have a local decomposition

$$
f(z)=h(z)+\overline{g(z)}
$$

with analytic $h, g$, but are themselves non-analytic in general. Several results on the number of zeros for special classes of harmonic mappings are known in

${ }^{*}$ TU Berlin, Department of Mathematics, MA 3-3, Straße des 17. Juni 136, 10623 Berlin, Germany. \{sete,zur\}@math.tu-berlin.de 
the literature, e.g., for harmonic polynomials, i.e., $f(z)=p(z)+\overline{q(z)}$, where $p$ and $q$ are analytic polynomials [44, 16, 9, 17], for rational harmonic mappings of the form $f(z)=r(z)-\bar{z}$, where $r$ is a rational function [14, 5, 21, 22, 35, 34, 18, 19, 37, or certain transcendental harmonic mappings [8, 4, 13, 20].

While the above publications have a theoretical focus, we are here interested in the numerical computation of the zeros of $f$. A single zero can be computed by an iterative root finder, e.g., Newton's method on $\mathbb{R}^{2}$ (see [36] for a convenient formulation for harmonic mappings). The computation of all zeros is much more challenging. Of course, one could focus on certain regions in the complex plane and run the root finder with multiple starting points. The problem with such an approach is, however, that in general the number of zeros of a harmonic mapping is not known a priori. In fact, even for harmonic polynomials $f(z)=p(z)-\bar{z}$ with $\operatorname{deg}(p)=n \geq 2$, the number of zeros can vary between $n$ and $3 n-2$, and each of these different numbers of zeros is attained by some harmonic polynomial; see [37, Thm. 5.4].

In this paper we present a continuation method to compute all zeros of a (non-degenerate) harmonic mapping, provided that the number of zeros is finite. We are not aware of any method for this problem in the literature that is specialized to harmonic mappings. Continuation is a general scheme that has been successfully applied to the numerical solution of systems of nonlinear equations (see, e.g., [31, Ch. 7.5] or [1]), and in particular for the solution of polynomial systems (see, e.g., [40] or [26]). Moreover, it is used in a wide range of applications, including mathematics for economics [12, Ch. 5], chemical physics [25] and astrophysics [33, Sect. 10.5]. In our continuation approach, the considered function changes only by a constant, which is sometimes called Newton homotopy. The overall idea is as follows. First we solve $f(z)=\eta_{1}$ for some $\eta_{1} \in \mathbb{C}$. We then construct a sequence $\eta_{2}, \eta_{3}, \ldots$ and solve $f(z)=\eta_{k+1}$ based on the solutions of $f(z)=\eta_{k}$. We let the sequence $\left(\eta_{k}\right)_{k}$ tend towards the origin and eventually, after finitely many steps, we end with $\eta_{n}=0$, so that solving $f(z)=\eta_{n}$ yields (numerically) all zeros of $f$.

Our method, which we call the transport of images method, works without any prior knowledge of the number of zeros or their approximate location in the complex plane. The main novelty is that we overcome two typical difficulties encountered in continuation methods.

1. Finding start points: To set up the method all solutions of $f(z)=\eta_{1}$ for some $\eta_{1} \in \mathbb{C}$ are required. In [37, Thm. 3.6] we proved that for sufficiently large $\left|\eta_{1}\right|$ all solutions of $f(z)=\eta_{1}$ are close to the poles of $f$, which are usually known. Then all solutions can be computed as in [36, Sect. 4].

2. Determine where and how the number of solutions changes: For harmonic mappings, the number of solutions of $f(z)=\eta_{k}$ depends on $\eta_{k} \in \mathbb{C}$; see, e.g., [37, Remark 5.5]. More precisely, the number of 
solutions of $f(z)=\eta_{k}$ and $f(z)=\eta_{k+1}$ differs by 2 if $\eta_{k}$ and $\eta_{k+1}$ are separated by a single arc of the caustics of $f$ (curves of the critical values); see [37. We deal with this effect as follows. For every step from $\eta_{k}$ to $\eta_{k+1}$ we construct a (minimal) set of points $S_{k+1}$, such that all solutions of $f(z)=\eta_{k+1}$ are obtained by applying Newton's method with initial points from $S_{k+1}$. This is based on results of Lyzzaik [23], Neumann [30] and the authors [37.

We prove that the transport of images method is guaranteed to compute all zeros of a non-degenerate harmonic mapping without singular zeros within a finite number of continuation steps.

Our numerical examples highlight three key features of our method: (1) It terminates with the correct number of zeros. (2) It is significantly faster than methods that are not problem adapted, such as Chebfun2 ${ }^{1}$. (3) It is highly accurate in terms of the residual. An easy-to-use MATLAB implementation ${ }^{2}$ is freely available online.

The name 'transport of images' method is borrowed from astrophysics. In [33, Sect. 10.5], the idea is roughly described in the context of gravitational lensing. There, the parameter $\eta$ models the position of a far away light source, and its lensed images are represented by the solutions of $f(z)=$ $\eta$ with a harmonic mapping $f$; see the surveys [15, 32, 3]. However, the description in [33] lacks many details and contains no further analysis.

Our paper is organized as follows. Relevant results on harmonic mappings are recalled in Section 2, In Section 3 we study the transport of images method and investigate the solution curves of $f(z)=\eta(t)$ depending on $t$. Key aspects of our implementation are discussed in Section 4. Section 5 contains several numerical examples that illustrate our method. We close with concluding remarks and a brief outlook in Section 6 .

\section{Preliminaries}

We briefly present relevant results for the computation of all zeros of harmonic mappings, following the lines of [37] and [23].

\subsection{Decomposition of harmonic mappings}

Let $\Omega \subseteq \mathbb{C}$ be open and connected. A harmonic mapping on $\Omega$ is a function $f: \Omega \rightarrow \mathbb{C}$ with $\Delta f=4 \partial_{\bar{z}} \partial_{z} f=0$, where $\partial_{z}$ and $\partial_{\bar{z}}$ denote the Wirtinger derivatives. If $f$ is harmonic in the open disk $D_{r}\left(z_{0}\right)=\left\{z \in \mathbb{C}:\left|z-z_{0}\right|<r\right\}$,

\footnotetext{
${ }^{1}$ Chebfun2, version of September 30, 2020, www.chebfun.org.

${ }^{2}$ Transport of images toolbox, https://github.com/transportofimages/
} 
it has a local decomposition

$$
f(z)=h(z)+\overline{g(z)}=\sum_{k=0}^{\infty} a_{k}\left(z-z_{0}\right)^{k}+\overline{\sum_{k=0}^{\infty} b_{k}\left(z-z_{0}\right)^{k}}, \quad z \in D_{r}\left(z_{0}\right),
$$

with analytic functions $h$ and $g$ in $D_{r}\left(z_{0}\right)$, which are unique up to an additive constant; see [7, p. 412] or [6, p. 7]. If $f$ is harmonic in the punctured disk $D=\left\{z \in \mathbb{C}: 0<\left|z-z_{0}\right|<r\right\}$, i.e., $z_{0}$ is an isolated singularity of $f$, then

$$
f(z)=\sum_{k=-\infty}^{\infty} a_{k}\left(z-z_{0}\right)^{k}+\overline{\sum_{k=-\infty}^{\infty} b_{k}\left(z-z_{0}\right)^{k}}+2 A \log \left|z-z_{0}\right|, \quad z \in D ;
$$

see [41, 10, 2]. The point $z_{0}$ is a pole of $f$ if $\lim _{z \rightarrow z_{0}} f(z)=\infty$, and an essential singularity if the limit does not exist in $\widehat{\mathbb{C}}=\mathbb{C} \cup\{\infty\}$; see [38, p. 44], [41, Def. 2.1] or [37, Def. 2.9]. It is possible that $f$ has a pole at $z_{0}$, but both series in (2.2) have an essential singularity at $z_{0}$, as the example $f(z)=z^{-2}+\cos (i / z)+\overline{\cos (i / z)}$ from [41, Ex. 2.3] shows. It is also possible that $f$ has an essential singularity at $z_{0}$, but both series have a pole at $z_{0}$, as shown by $f(z)=z^{-1}+\bar{z}^{-1}$.

The next proposition is the analog of the well-known facts that a function holomorphic on $\widehat{\mathbb{C}}$ is constant and a function meromorphic on $\widehat{\mathbb{C}}$ is rational.

Proposition 2.1. A harmonic mapping $f$ on $\widehat{\mathbb{C}} \backslash\left\{z_{1}, \ldots, z_{m}\right\}$ with poles at $z_{1}, \ldots, z_{m} \in \widehat{\mathbb{C}}$, at which the principal parts of both series in the decomposition 2.2. have only finitely many terms, has the form

$$
f(z)=r(z)+\overline{s(z)}+\sum_{j=1}^{m} 2 A_{j} \log \left|z-z_{j}\right|
$$

where $A_{1}, \ldots, A_{m} \in \mathbb{C}$, and $r$ and $s$ are rational functions that can have poles only at $z_{1}, \ldots, z_{m}$. If some $z_{j}=\infty$, then $\log |z|$ replaces $\log \left|z-z_{j}\right|$. In particular, if $f$ is harmonic on $\widehat{\mathbb{C}}$, then $f$ is constant.

Proof. First, let $f$ be a harmonic mapping on $\widehat{\mathbb{C}}$. Then $\partial_{z} f$ is analytic on $\widehat{\mathbb{C}}$ and thus constant [43, Thm. 3.5.8]. Let $h^{\prime}=\partial_{z} f$, then $h(z)=a_{1} z+a_{0}$ is analytic. Let $g=\overline{f-h}$. Since $\partial_{\bar{z}} g=\overline{\partial_{z} f-h^{\prime}}=0, g$ is analytic on $\widehat{\mathbb{C}}$ and hence constant, say $g(z)=b_{0}$, and we have $f(z)=a_{1} z+a_{0}+\bar{b}_{0}$. Since $f$ is harmonic at $\infty$ we have $a_{1}=0$ and $f$ is constant. Next, let $f$ be harmonic with poles at $z_{1}, \ldots, z_{m}$. If $z_{j} \neq \infty$ the decomposition (2.2) has the form

$$
f(z)=\sum_{k=-n}^{\infty}\left(a_{k}\left(z-z_{j}\right)^{k}+\overline{b_{k}\left(z-z_{j}\right)^{k}}\right)+2 A_{j} \log \left|z-z_{j}\right|
$$

and we set $f_{j}(z)=\sum_{k=-n}^{-1}\left(a_{k}\left(z-z_{j}\right)^{k}+\overline{b_{k}\left(z-z_{j}\right)^{k}}\right)+2 A_{j} \log \left|z-z_{j}\right|$ and similarly if $z_{j}=\infty$. Then $f-f_{1}-\ldots-f_{m}$ has removable singularities at $z_{1}, \ldots, z_{m}$, and can be extended to a harmonic mapping in $\widehat{\mathbb{C}}$ (see [37, Sect. 2.2], or also [11, Thm. 15.3d]), which is constant. 


\subsection{Critical set and caustics of harmonic mappings}

The critical points of a harmonic mapping $f: \Omega \rightarrow \mathbb{C}$, i.e., the points where the Jacobian of $f$,

$$
J_{f}(z)=\left|\partial_{z} f(z)\right|^{2}-\left|\partial_{\bar{z}} f(z)\right|^{2},
$$

vanishes, form the critical set of $f$,

$$
\mathcal{C}=\left\{z \in \Omega: J_{f}(z)=0\right\} .
$$

By Lewy's theorem [6, p. 20] $f$ is locally univalent at $z \in \Omega$ if and only if $z \notin \mathcal{C}$. Here and in the following we assume that $\partial_{z} f$ and $\partial_{\bar{z}} f$ do not vanish identically. (Otherwise $f$ would be analytic or anti-analytic, which is not the focus of this paper.) Then $\partial_{z} f$ has only isolated zeros in $\Omega$, and the critical set consists of isolated points and a level set of the second complex dilatation of $f$,

$$
\omega(z)=\frac{\overline{\partial_{\bar{z}} f(z)}}{\partial_{z} f(z)},
$$

which is a meromorphic function in $\Omega$. We assume that removable singularities of $\omega$ in $\Omega$ are removed. Let

$$
\begin{aligned}
\mathcal{M} & =\{z \in \mathcal{C}:|\omega(z)| \neq 1\} \\
& =\left\{z \in \Omega: \partial_{z} f(z)=\partial_{\bar{z}} f(z)=0 \text { and } \lim _{\zeta \rightarrow z}|\omega(\zeta)| \neq 1\right\},
\end{aligned}
$$

see [37, Sect. 2.1], which consists of isolated points of $\mathcal{C}$ [23, Lem. 2.2]. Then

$$
\mathcal{C} \backslash \mathcal{M}=\{z \in \Omega:|\omega(z)|=1\}
$$

is a level set of $\omega$. If $J_{f} \not \equiv 0$, then $\mathcal{C} \backslash \mathcal{M}$ consists of analytic curves. These intersect in $z \in \mathcal{C} \backslash \mathcal{M}$ if, and only if, $\omega^{\prime}(z)=0$. At $z \in \mathcal{C} \backslash \mathcal{M}$ with $\omega^{\prime}(z) \neq 0$, the equation

$$
\omega(\gamma(t))=e^{i t}, \quad t \in I \subseteq \mathbb{R},
$$

implicitly defines a local analytic parametrization $z=\gamma(t)$ of $\mathcal{C} \backslash \mathcal{M}$.

Definition 2.2. We call the set of critical values of $f$, i.e., $f(\mathcal{C})$, the caustics or the set of caustic points. The caustics induce a partition of $\mathbb{C} \backslash f(\mathcal{C})$, and we call a connected component $A \subseteq \mathbb{C} \backslash f(\mathcal{C})$ with $\partial A \subseteq f(\mathcal{C})$ a caustic tile.

The next lemma characterizes a tangent vector to the caustics; see [37, Lem. 2.1] or [23, Lem. 2.3].

Lemma 2.3. Let $f$ be a harmonic mapping, $z_{0} \in \mathcal{C} \backslash \mathcal{M}$ with $\omega^{\prime}\left(z_{0}\right) \neq 0$ and let $z_{0}=\gamma\left(t_{0}\right)$ with the parametrization 2.10). Then $f \circ \gamma$ is a parametrization of a caustic and the corresponding tangent vector at $f\left(z_{0}\right)$ is

$$
\tau\left(t_{0}\right)=\frac{d}{d t}(f \circ \gamma)\left(t_{0}\right)=e^{-i t_{0} / 2} \psi\left(t_{0}\right)
$$


with

$$
\psi(t)=2 \operatorname{Re}\left(e^{i t / 2} \partial_{z} f(\gamma(t)) \gamma^{\prime}(t)\right) .
$$

In particular, the rate of change of the argument of the tangent vector is

$$
\left.\frac{d}{d t} \arg (\tau(t))\right|_{t=t_{0}}=-\frac{1}{2}
$$

at points where $\psi\left(t_{0}\right) \neq 0$, i.e., the curvature of the caustics is constant with respect to the parametrization $f \circ \gamma$. Moreover, $\psi$ has either only finitely many zeros, or is identically zero, in which case $f \circ \gamma$ is constant.

Definition 2.4 ([37, Def. 2.2]). In the notation of Lemma 2.3 we call $f\left(\gamma\left(t_{0}\right)\right)$ a fold caustic point or simply a fold, if the tangent $\tau\left(t_{0}\right)$ is non-zero, i.e., $\psi\left(t_{0}\right) \neq 0$, and a cusp if $\psi$ has a zero with a sign change at $t_{0}$.

This classification of caustic points is not complete but sufficient for our needs. Fold points form open arcs of the caustic curves, since $\psi$ is continuous. At a cusp the argument of the tangent vector changes by $+\pi$. The cusps, the curvature of the caustics and the four caustic tiles are apparent in Figure 1 (left).

\subsection{Pre-images under non-degenerate harmonic mappings}

The position of $\eta \in \mathbb{C}$ with respect to the caustics affects the number of pre-images of $\eta$ under $f$. The following wide class of harmonic mappings was introduced in [37, Def. 3.1].

Definition 2.5. We call a harmonic mapping $f$ non-degenerate on $\widehat{\mathbb{C}}$ if

1. $f$ is defined in $\widehat{\mathbb{C}}$ except at finitely many poles $z_{1}, \ldots, z_{m} \in \widehat{\mathbb{C}}$,

2. at each pole $z_{j} \in \mathbb{C}$ of $f$, the decomposition 2.2 of $f$ has the form

$$
f(z)=\sum_{k=-n}^{\infty} a_{k}\left(z-z_{j}\right)^{k}+\overline{\sum_{k=-n}^{\infty} b_{k}\left(z-z_{j}\right)^{k}}+2 A_{j} \log \left|z-z_{j}\right|
$$

with $n \geq 1$ and $\left|a_{-n}\right| \neq\left|b_{-n}\right|$, and if $z_{j}=\infty$ is a pole of $f$, then

$$
f(z)=\sum_{k=-\infty}^{n} a_{k} z^{k}+\overline{\sum_{k=-\infty}^{n} b_{k} z^{k}}+2 A_{j} \log |z|, \quad \text { for }|z|>R,
$$

with $n \geq 1$ and $\left|a_{n}\right| \neq\left|b_{n}\right|$, and $R>0$,

3. the critical set of $f$ is bounded in $\mathbb{C}$. 
The intensively studied (see references in the introduction) rational harmonic mappings $r(z)-\bar{z}$ and harmonic polynomials $p(z)+\overline{q(z)}$ are nondegenerate if, and only if, $\lim _{z \rightarrow \infty}|r(z) / z| \neq 1$ and $\lim _{z \rightarrow \infty}|p(z) / q(z)| \neq 1$, respectively, since $\infty$ is the only pole of $p(z), q(z)$ and $z$.

A non-degenerate harmonic mapping $f$ has a global decomposition 2.3, and

$$
\partial_{z} f(z)=r^{\prime}(z)+\sum_{j=1}^{m} \frac{A_{j}}{z-z_{j}}, \quad \overline{\partial_{z} f(z)}=s^{\prime}(z)+\sum_{j=1}^{m} \frac{\bar{A}_{j}}{z-z_{j}},
$$

are rational functions. By item 2., the poles are not accumulation points of the critical set [41, Lem. 2.2, 2.3]. Hence, $\mathcal{C}$ and $f(\mathcal{C})$ are compact sets in C. Item 3. implies that $J_{f} \not \equiv 0$. If $\partial_{z} f \equiv 0$, then $\overline{\partial_{\bar{z}} f} \not \equiv 0$ (since $J_{f} \not \equiv 0$ ), and $\mathcal{C}$ consists of the finitely many zeros of the rational function $\overline{\partial_{\bar{z}} f}$. If $\partial_{z} f \not \equiv 0$, then $\omega=\overline{\partial_{\bar{z}} f} / \partial_{z} f$ is rational, $\mathcal{M}$ has only finitely many points, and $\mathcal{C} \backslash \mathcal{M}$ consists of the $\operatorname{deg}(\omega)$ many pre-images of the unit circle under $\omega$. We can parametrize $\mathcal{C} \backslash \mathcal{M}$ with analytic closed curves according to 2.10), such that every $z \in \mathcal{C} \backslash \mathcal{M}$ with $\omega^{\prime}(z) \neq 0$ belongs to exactly one curve; see [37, Seect. 3.1]. We call these curves the critical curves of $f$ and denote the set of all of them by crit. The critical curves and their images under $f$ both have finite total length since their parametrization is piecewise $C^{\infty}$.

Let $P(f)=n_{1}+\ldots+n_{m}$ be the sum of the orders of the poles $z_{1}, \ldots, z_{m}$ in 2.14 or 2.15. Then $N_{\eta}(f)=|\{z \in \widehat{\mathbb{C}}: f(z)=\eta\}|$, the number of pre-images of $\eta$ under $f$, relates to $P(f)$ and the winding number $n(f \circ \gamma ; \eta)$ of the caustics about $\eta$ as follows.

Theorem 2.6 ([37, Thms. 3.4, 3.7]). Let $f$ be a non-degenerate harmonic mapping on $\widehat{\mathbb{C}}$. Then for a point $\eta \in \mathbb{C} \backslash f(\mathcal{C})$ we have

$$
N_{\eta}(f)=P(f)+2 \sum_{\gamma \in \text { crit }} n(f \circ \gamma ; \eta)
$$

In particular, the number of pre-images of $\eta \in \mathbb{C} \backslash f(\mathcal{C})$ under $f$ is finite. Moreover, if $\eta_{1}, \eta_{2} \in \mathbb{C} \backslash f(\mathcal{C})$ then

$$
N_{\eta_{2}}(f)=N_{\eta_{1}}(f)+2 \sum_{\gamma \in \text { crit }}\left(n\left(f \circ \gamma ; \eta_{2}\right)-n\left(f \circ \gamma ; \eta_{1}\right)\right),
$$

and we have:

1. If $\eta_{1}$ and $\eta_{2}$ are in the same caustic tile then the number of pre-images under $f$ is the same, i.e., $N_{\eta_{2}}(f)=N_{\eta_{1}}(f)$.

2. If $\eta_{1}$ and $\eta_{2}$ are separated by a single caustic arc $f \circ \gamma$ then the number of pre-images under $f$ differs by 2, i.e., $N_{\eta_{2}}(f)=N_{\eta_{1}}(f) \pm 2$. 


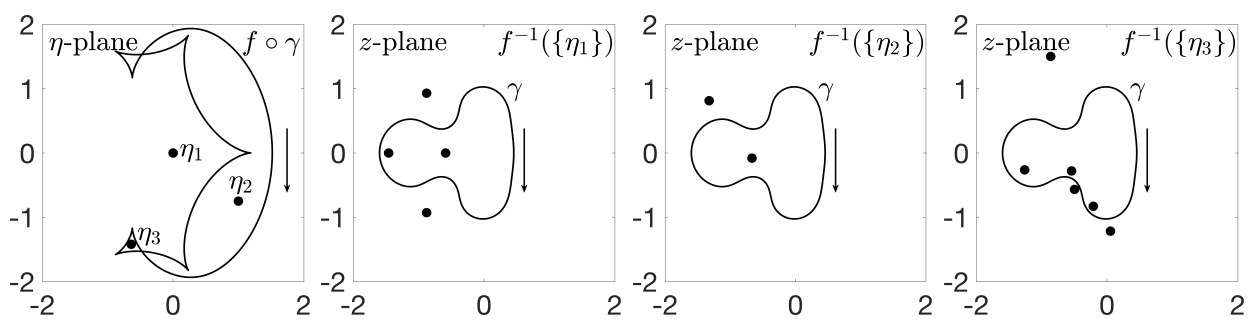

Figure 1: Harmonic mapping (2.19). $\eta$-plane: the caustic $f \circ \gamma$ of $f$ and the points $\eta_{j}$. $z$-plane: pre-images of $\eta_{j}$ under $f$. The arrows indicate the orientation of the critical curve $\gamma$ and the caustic $f \circ \gamma$.

Example 2.7 ([37, Ex. 3.10]). We study the non-degenerate harmonic mapping

$$
f(z)=z^{2}+\overline{z^{-1}+(z+1)^{-1}}+2 \log |z| ;
$$

see Figure 1. By Lemma 2.3 the curvature of a caustic is always negative. Consequently, the winding numbers of the only caustic $f \circ \gamma$ about $\eta_{1}, \eta_{2}$ and $\eta_{3}$ are $0,-1$ and 1 respectively. By Theorem 2.6 we have

$$
\begin{aligned}
& N_{\eta_{1}}(f)=P(f)+2 n\left(f \circ \gamma ; \eta_{1}\right)=4+0=4, \\
& N_{\eta_{2}}(f)=N_{\eta_{1}}(f)+2\left(n\left(f \circ \gamma ; \eta_{2}\right)-n\left(f \circ \gamma ; \eta_{1}\right)\right)=4-2=2, \\
& N_{\eta_{3}}(f)=N_{\eta_{1}}(f)+2\left(n\left(f \circ \gamma ; \eta_{3}\right)-n\left(f \circ \gamma ; \eta_{1}\right)\right)=4+2=6,
\end{aligned}
$$

as we see in Figure 1 .

\section{The transport of images method}

We compute all zeros of a non-degenerate harmonic mapping $f$ by continuation, provided that no zero of $f$ is singular, or equivalently, if 0 belongs to the caustics of $f$. A zero $z_{0}$ is singular, if $J_{f}\left(z_{0}\right)=0$ holds. Recall from the introduction that we successively compute all solutions of $f(z)=\eta_{k}$ for a sequence $\eta_{1}, \eta_{2}, \ldots$ that tends to zero. During this procedure the number of solutions may change, depending on the positions of the points $\eta_{k}$ relative to the caustics of $f$; see Theorem 2.6. We start by computing all solutions of $f(z)=\eta_{1}$ for sufficiently large $\left|\eta_{1}\right|$ from the local decomposition 2.2 of $f$ near the poles. Given all solutions of $f(z)=\eta_{k}$ we determine an $\eta_{k+1} \in \mathbb{C}$ and a set $S_{k+1} \subset \mathbb{C}$ (prediction) such that the following holds: (1) for every solution $z_{*} \in \mathbb{C}$ of $f(z)=\eta_{k+1}$ there exists a $z_{0} \in S_{k+1}$ such that the sequence $\left(z_{j}\right)_{j \in \mathbb{N}}$ of Newton iterates for $f-\eta_{k+1}$ converges to $z_{*}$ (correction); (2) the number of solutions of $f(z)=\eta_{k+1}$ coincides with the number of elements in $S_{k+1}$, i.e., $S_{k+1}$ is minimal. When this prediction-correction scheme reaches $\eta_{n}=0$ we obtain all zeros of $f$. This leads to the following algorithm. 


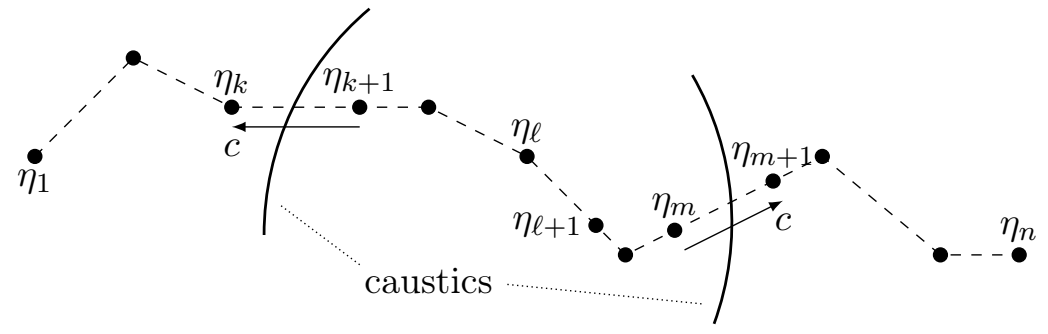

Figure 2: A path from $\eta_{1}$ to $\eta_{n}$ with the three kinds of steps in the transport of images method: (1) $f(z)=\eta_{k+1}$ has two solutions fewer than $f(z)=\eta_{k}$, (2) the number of solutions for $\eta_{\ell}$ and $\eta_{\ell+1}$ coincides and (3) $f(z)=\eta_{m+1}$ has two solutions more than $f(z)=\eta_{m}$.

\section{The transport of images method}

1. Initial phase: compute all solutions of $f(z)=\eta_{1}$.

2. Transport phase: while $\eta_{k} \neq 0$ do

(a) Prediction: choose $\eta_{k+1} \in \mathbb{C}$ and construct a minimal set of initial points $S_{k+1}$ from the solutions of $f(z)=\eta_{k}$ and from the caustics.

(b) Correction: apply Newton's method to $f-\eta_{k+1}$ with the set of initial points $S_{k+1}$ to get all solutions of $f(z)=\eta_{k+1}$.

The points $\eta_{1}, \eta_{2}, \ldots, \eta_{n}=0$ conceptually form a path in $\mathbb{C}$ (see Figure2). From this perspective the transport of images method discretizes the tracing of the solution curves of $f(z)=\eta(t)$, where the right hand side is a path $\eta:[a, b] \rightarrow \mathbb{C}$. We describe the homotopy curves for a general harmonic mapping in Section 3.4 in full detail.

\subsection{Newton's method as corrector}

For a harmonic mapping $f: \Omega \rightarrow \mathbb{C}$ the harmonic Newton map is

$$
H_{f}: \Omega \backslash \mathcal{C} \rightarrow \mathbb{C}, \quad H_{f}(z)=z-\frac{\overline{h^{\prime}(z)} f(z)-\overline{g^{\prime}(z) f(z)}}{\left|h^{\prime}(z)\right|^{2}-\left|g^{\prime}(z)\right|^{2}},
$$

where $f=h+\bar{g}$ is a local decomposition (2.1) and the harmonic Newton iteration with initial point $z_{0} \in \Omega \backslash \mathcal{C}$ is

$$
z_{k}=H_{f}\left(z_{k-1}\right)=H_{f}^{k}\left(z_{0}\right), \quad k \geq 1 .
$$

We write, as usual, $H_{f}^{k}(z)=H_{f}\left(H_{f}^{k-1}(z)\right)$ for $k \geq 1$ and $H_{f}^{0}(z)=z$. The iteration (3.2) is a complex formulation of the classical Newton iteration in $\mathbb{R}^{2}$ [36, Sect. 3.1] and hence inherits all its properties, e.g., local quadratic convergence. 
To compute solutions of $f(z)=\eta$ with $\eta \in \mathbb{C}$ we apply the iteration 3.2 to $f-\eta$. The limit of the harmonic Newton iteration with initial point $z_{0}$,

$$
H_{f-\eta}^{\infty}\left(z_{0}\right)=\lim _{k \rightarrow \infty} H_{f-\eta}^{k}\left(z_{0}\right),
$$

exists for all $z_{0}$ in a neighborhood of a solution $z_{*} \notin \mathcal{C}$ of $f(z)=\eta$ by the local convergence property of Newton's method. If $z_{*}=H_{f-\eta}^{\infty}\left(z_{0}\right)$ holds we say that $z_{*}$ attracts $z_{0}$ under $H_{f-\eta}$ or that $z_{0}$ is attracted by $z_{*}$ under $H_{f-\eta}$.

Definition 3.1. For a harmonic mapping $f$ we call a set $S$ a (minimal) prediction set of $f^{-1}(\{\eta\})$ if every solution of $f(z)=\eta$ attracts exactly one point in $S$ under $H_{f-\eta}$, i.e., if $H_{f-\eta}^{\infty}(S)=f^{-1}(\{\eta\})$, and $|S|=\left|f^{-1}(\{\eta\})\right|$, where $|X|$ denotes the cardinality of a set $X$.

For a prediction set $S$ the map $H_{f-\eta}^{\infty}$ is a bijection from $S$ to $f^{-1}(\{\eta\})$. In this sense every point $z_{0} \in S$ represents exactly one solution of $f(z)=\eta$. In [39] the point $z_{0}$ is called an approximate zero of $f-\eta$ if the Newton iterates additionally satisfy $\left|z_{k}-z_{k-1}\right| \leq\left(\frac{1}{2}\right)^{2^{k-1}-1}\left|z_{1}-z_{0}\right|$.

\subsection{Initial phase}

For non-degenerate harmonic mappings the initial phase can be realized as follows. For sufficiently large $|\eta|$ all solutions of $f(z)=\eta$ are close to the poles of $f$ and, in the notation (2.14) or 2.15), there are exactly $n$ solutions close to the pole $z_{j}$ [37, Thm. 3.6]. We compute these solutions with the harmonic Newton iteration and the initial points from the next theorem. A similar result holds if $\infty$ is a pole of $f$, generalizing [36, Cor. 4.5]. All initial points together give a prediction set of $f^{-1}(\{\eta\})$.

Theorem 3.2. Let $f$ be harmonic in $D=\left\{z \in \mathbb{C}: 0<\left|z-z_{0}\right|<r\right\}$ with

$$
f(z)=\sum_{k=-n}^{\infty} a_{k}\left(z-z_{0}\right)^{k}+\overline{\sum_{k=-n}^{\infty} b_{k}\left(z-z_{0}\right)^{k}}+2 A \log \left|z-z_{0}\right|, \quad z \in D,
$$

where $n \geq 1$ and $\left|a_{-n}\right| \neq\left|b_{-n}\right|$. Suppose that $c=\eta-\left(a_{0}+\bar{b}_{0}\right) \neq 0$ and let $\zeta_{1}, \ldots, \zeta_{n}$ be the $n$ solutions of

$$
\left(\zeta-z_{0}\right)^{n}=\frac{\left|a_{-n}\right|^{2}-\left|b_{-n}\right|^{2}}{\bar{a}_{-n} c-\bar{b}_{-n} \bar{c}} .
$$

We then have for sufficiently large $|c|$, i.e., for sufficiently large $|\eta|$ :

1. There exist exactly $n$ solutions of $f(z)=\eta$ near $z_{0}$.

2. The set $S=\left\{\zeta_{1}, \ldots, \zeta_{n}\right\}$ is a prediction set for the solutions in 1 .

Proof. If $A=0$ then $f-\eta$ has $n$ distinct zeros near $z_{0}$, which satisfy 2 . by [36, Thm. 4.3]. For $A \neq 0$ the proof closely follows the proof of [36, Thm. 4.3] if one replaces $h^{\prime}$ by $\partial_{z} f$, and $g^{\prime}$ by $\overline{\partial_{\bar{z}} f}$. As in the proof of [37, Thm. 3.6], Rouché's theorem (e.g. [35, Thm. 2.3]) implies that $f-\eta$ cannot have more than $n$ zeros in a neighborhood of $z_{0}$. 


\subsection{Transport phase}

We analyze the prediction-correction scheme of the transport phase and prove that the transport phase can be realized with a finite number of steps. We begin with a single step and investigate how all solutions of $f(z)=\eta_{2}$ can be determined, given all solutions of $f(z)=\eta_{1}$. First we prove that a solution of $f(z)=\eta_{1}$ that is not in $\mathcal{C}$ is attracted by a solution of $f(z)=\eta_{2}$ under the harmonic Newton map $H_{f-\eta_{2}}$ if $\left|\eta_{2}-\eta_{1}\right|$ is small enough.

Lemma 3.3. Let $f: \Omega \rightarrow \mathbb{C}$ be a harmonic mapping, $z_{0} \in \Omega \backslash \mathcal{C}$ and $\eta_{1}=f\left(z_{0}\right)$. Then there exist $\varepsilon>0$ and $\delta>0$ such that for each $\eta_{2} \in D_{\varepsilon}\left(\eta_{1}\right)$ there exists a unique $z_{*} \in D_{\delta}\left(z_{0}\right)$ with $f\left(z_{*}\right)=\eta_{2}$. Moreover, $z_{*}$ attracts $z_{0}$ under the harmonic Newton map $H_{f-\eta_{2}}$, i.e., $H_{f-\eta_{2}}^{\infty}\left(z_{0}\right)=z_{*}$.

Proof. Let $\zeta \in \Omega \backslash \mathcal{C}$ and consider $f-f(\zeta)$. The Newton-Kantorovich theorem yields a radius $\rho(\zeta)>0$ so that $\zeta$ attracts all points in $D_{\rho(\zeta)}(\zeta)$ under the harmonic Newton map of $f-f(\zeta)$; see [36. Thm. 2.2, Sect. 4] and references therein. (The basin of attraction of $\zeta$ might be larger.) Note that $\rho$ depends continuously on $\zeta$.

There exist open neighborhoods $U \subseteq \Omega \backslash \mathcal{C}$ of $z_{0}$ and $V$ of $\eta_{1}$ such that $f: U \rightarrow V$ is a diffeomorphism (inverse function theorem). Let $r>0$ with $K=\overline{D_{r}\left(z_{0}\right)} \subseteq U$ and let $m=\min _{z \in K} \rho(z)$. Then $m>0$ since $K$ is compact and $\rho$ is continuous with $\rho(z)>0$ for $z \in U$. Let $0<\delta \leq \min \{r, m\}$. Then $z_{*} \in D_{\delta}\left(z_{0}\right)$ satisfies $\left|z_{*}-z_{0}\right|<\delta \leq m \leq \rho\left(z_{*}\right)$, hence $H_{f-f\left(z_{*}\right)}^{\infty}\left(z_{0}\right)=z_{*}$. Finally, there exists $\varepsilon>0$ with $D_{\varepsilon}\left(\eta_{1}\right) \subseteq f\left(D_{\delta}\left(z_{0}\right)\right)$.

By Theorem 2.6, two points in the same caustic tile have the same number of pre-images. Moreover, $\eta \in \mathbb{C} \backslash f(\mathcal{C})$ has only finitely many pre-images, and these are in $\widehat{\mathbb{C}} \backslash \mathcal{C}$. Thus, we can apply Lemma 3.3 to all solutions simultaneously to obtain the next theorem.

Theorem 3.4. Let $f$ be a non-degenerate harmonic mapping on $\widehat{\mathbb{C}}$ and let $\eta_{1} \in \mathbb{C} \backslash f(\mathcal{C})$. Then there exists an $\varepsilon>0$ such that $S=f^{-1}\left(\left\{\eta_{1}\right\}\right)$ is a prediction set of $f^{-1}\left(\left\{\eta_{2}\right\}\right)$ for all $\eta_{2} \in D_{\varepsilon}\left(\eta_{1}\right) \subseteq \mathbb{C} \backslash f(\mathcal{C})$.

By Theorem 2.6, the number of pre-images of $\eta_{1}, \eta_{2}$ differs by 2 if $\eta_{1}, \eta_{2}$ are in adjacent caustic tiles separated by a single caustic arc. The two additional solutions can be computed with the next theorem, provided that the step from $\eta_{1}$ to $\eta_{2}$ crosses the caustics in a specific direction.

Theorem 3.5 ([37, Thm. 4.2]). Let $f: \Omega \rightarrow \mathbb{C}$ be a harmonic mapping and $z_{0} \in \mathcal{C} \backslash \mathcal{M}$, such that $\eta=f\left(z_{0}\right)$ is a fold caustic point. Moreover, let

$$
f(z)=\sum_{k=0}^{\infty} a_{k}\left(z-z_{0}\right)^{k}+\overline{\sum_{k=0}^{\infty} b_{k}\left(z-z_{0}\right)^{k}} \quad \text { and } c=-\left(\frac{a_{2} \bar{b}_{1}}{a_{1}}+\frac{\bar{b}_{2} a_{1}}{\bar{b}_{1}}\right) .
$$

Then, for each sufficiently small $\varepsilon>0$, there exists a $\delta>0$ such that for all $0<t<\delta$ : 
1. $f(z)=\eta-t c$ has no solution in $D_{\varepsilon}\left(z_{0}\right)$,

2. $f(z)=\eta$ has exactly one solution in $D_{\varepsilon}\left(z_{0}\right)$,

3. $f(z)=\eta+t c$ has exactly two solutions in $D_{\varepsilon}\left(z_{0}\right)$.

Moreover, each solution in 3. attracts one of the points

$$
z_{ \pm}=z_{0} \pm i \sqrt{t \bar{b}_{1} / a_{1}}
$$

under the harmonic Newton map $H_{f-(\eta+t c)}$ if $t>0$ is sufficiently small.

Note that $f$ is light (i.e., $f^{-1}(\{\eta\})$ is either totally disconnected or empty for all $\eta \in \mathbb{C}$ ) in a neighborhood of $z_{0}$ if $f\left(z_{0}\right)$ is a fold [37, Rem. 2.3]; hence we omit this assumption from [36, Thm. 4.2]. We emphasize that $c$ is not necessarily a normal vector to the caustics.

With Theorem 3.5, we construct a prediction set of $f^{-1}\left(\left\{\eta_{2}\right\}\right)$ if the step from $\eta_{1}$ to $\eta_{2}$ crosses a caustic at a simple fold $\eta$, i.e., $\left|f^{-1}(\{\eta\}) \cap \mathcal{C}\right|=1$.

Theorem 3.6. Let $f$ be a non-degenerate harmonic mapping on $\widehat{\mathbb{C}}$. Let $\eta_{1}, \eta_{2} \in \mathbb{C} \backslash f(\mathcal{C})$ such that there exists exactly one simple fold $\eta$ and no other caustic point on the line segment from $\eta_{1}$ to $\eta_{2}$, and such that $\arg \left(\eta_{2}-\right.$ $\left.\eta_{1}\right)=\arg ( \pm c)$, where $c$ is defined as in Theorem 3.5. Then for small enough $\left|\eta_{2}-\eta_{1}\right|$

$$
S= \begin{cases}f^{-1}\left(\left\{\eta_{1}\right\}\right) \cup\left\{z_{ \pm}\right\}, & \text {if } \arg \left(\eta_{2}-\eta_{1}\right)=\arg (+c), \\ f^{-1}\left(\left\{\eta_{1}\right\}\right) \backslash H_{f-\eta_{1}}^{\infty}\left(\left\{z_{ \pm}\right\}\right), & \text {if } \arg \left(\eta_{2}-\eta_{1}\right)=\arg (-c),\end{cases}
$$

is a prediction set of $f^{-1}\left(\left\{\eta_{2}\right\}\right)$, with $z_{ \pm}$from 3.6 .

Proof. Let $\eta=f\left(z_{0}\right)$ be the unique simple fold on the line segment between $\eta_{1}$ and $\eta_{2}$. The step from $\eta_{1}$ to $\eta_{2}$ produces either two additional or two fewer solutions in a neighborhood of $z_{0}$, depending on $c$; see Theorem 3.5 . The global number of solutions changes accordingly; see Theorem 2.6.

In the case of two additional solutions, i.e., if $\arg \left(\eta_{2}-\eta_{1}\right)=\arg (+c)$, the set $S$ contains the solutions of $f(z)=\eta_{1}$, and the points $z_{ \pm}$, hence $|S|=\left|f^{-1}\left(\left\{\eta_{2}\right\}\right)\right|$ for sufficiently small $\left|\eta_{2}-\eta_{1}\right|$. Let $t_{1}, t_{2}>0$ such that $\eta_{1}=\eta-t_{1} c$ and $\eta_{2}=\eta+t_{2} c$. In particular, we have $t_{j} \leq\left|\left(\eta_{2}-\eta_{1}\right) / c\right|$. In the disk $D_{\varepsilon}\left(z_{0}\right)$ from Theorem 3.5, $f(z)=\eta_{1}$ has no solution and $f(z)=\eta_{2}$ has exactly two solutions, which attract $z_{+}$and $z_{-}$, respectively, if $\left|\eta_{2}-\eta_{1}\right|$ and hence $t_{2}$ are small enough. Since $\eta_{1}$ is not a caustic point, all solutions of $f(z)=\eta_{1}$ are not in $\mathcal{C}$. Hence, every solution of $f(z)=\eta_{2}$ outside $D_{\varepsilon}\left(z_{0}\right)$ attracts exactly one solution of $f(z)=\eta_{1}$ by Lemma 3.3 , if $\left|\eta_{2}-\eta_{1}\right|$ is sufficiently small. Together, $S$ is a prediction set of $f^{-1}\left(\left\{\eta_{2}\right\}\right)$.

In the case of two fewer solutions, i.e., if $\arg \left(\eta_{2}-\eta_{1}\right)=\arg (-c)$, we remove two points from $f^{-1}\left(\left\{\eta_{1}\right\}\right)$ to obtain a prediction set. To determine 

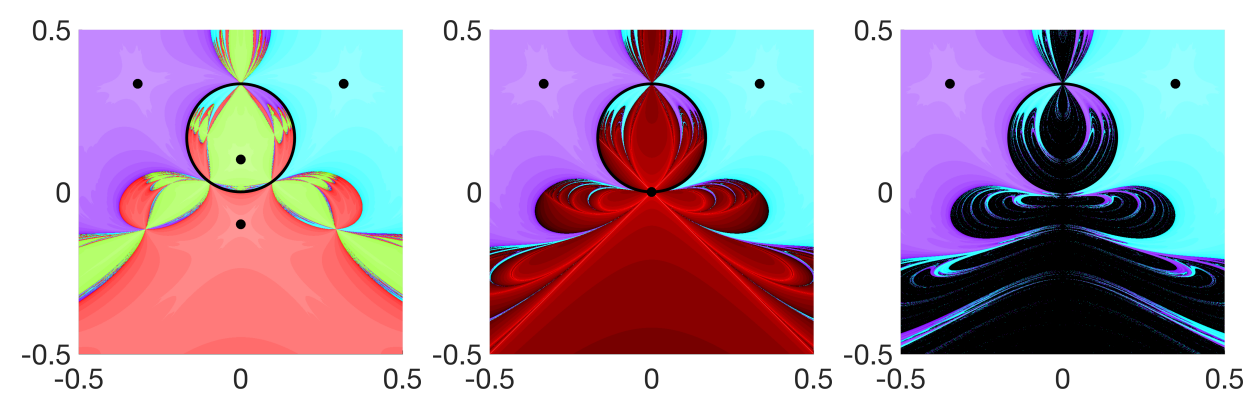

Figure 3: Solutions (black dots) of $f(z)=z+2 i z+\overline{z+i z^{2}}=t c$ with basins of attraction for $z_{0}=0, c=-i$ and $t=0.01, t=0, t=-0.01$ (from left to right); see Remark 3.8. The black circle is the critical set of $f$.

these points we consider the reversed step from $\eta_{2}$ to $\eta_{1}$. This step gives the two additional solutions $H_{f-\eta_{1}}^{\infty}\left(z_{ \pm}\right)$, which we remove from $f^{-1}\left(\left\{\eta_{1}\right\}\right)$ to get $S$. Each solution of $f(z)=\eta_{2}$ attracts exactly one point in $S$, as above.

Remark 3.7. Let $\eta \in f(\mathcal{C})$ be a (multiple) fold caustic point, i.e., for each $z_{j} \in\left\{z_{1}, \ldots, z_{m}\right\}=f^{-1}(\{\eta\}) \cap \mathcal{C}$ the tangent $\tau_{j}$ from (2.11) is nonzero. Then, for every $d \in \mathbb{C}$ with $\operatorname{Im}\left(\bar{\tau}_{j} d\right) \neq 0$ for $j=1, \ldots, m$, the effect of Theorem 3.5 happens at all points $z_{1}, \ldots, z_{m}$ simultaneously. By [37, Rem. 4.3], $f(z)=\eta+t d$ has 2 solutions in $D_{\varepsilon}\left(z_{j}\right)$ and $f(z)=\eta-t d$ has no solutions in $D_{\varepsilon}\left(z_{j}\right)$ if $\operatorname{Im}\left(\bar{\tau}_{j} d\right)>0$ and $t>0$ is sufficiently small. If $\operatorname{Im}\left(\bar{\tau}_{j} d\right)<$ 0 then $f(z)=\eta \pm t d$ swap their roles. However, it is not guaranteed that the additional solutions attract the points $z_{ \pm}\left(z_{j}\right)$ if $\arg (d) \neq \arg \left( \pm c\left(z_{j}\right)\right)$, with $z_{ \pm}$and $c$ from Theorem 3.5 .

Remark 3.8. The basins of attraction of the two solutions of $f(z)=\eta+t c$ in $D_{\varepsilon}\left(z_{0}\right)(t>0)$ merge $(t=0)$ and disappear $(t<0)$ with the solutions. This is illustrated in Figure 3 . Points that are attracted by the same solution have the same color. The darker the shading, the more iterations are needed until (numerical) convergence. Points where the iteration does not converge (numerically) are colored in black. This highlights why it is important to remove the two points $H_{f-\eta_{1}}^{\infty}\left(z_{ \pm}\right)$from $f^{-1}\left(\left\{\eta_{1}\right\}\right)$ in 3.7) for practical computations.

We illustrate Theorems 3.4 and 3.6 in Figure 4 . First, consider the step from $\eta_{1}$ to $\eta_{2}$ and the dynamics of $H_{f-\eta_{2}}$ (middle plot). Every basin of attraction contains exactly one element of $S=f^{-1}\left(\left\{\eta_{1}\right\}\right)$, i.e., $S$ is a prediction set of $f^{-1}\left(\left\{\eta_{2}\right\}\right)$. Some elements in $S$ are close to the boundary of the respective basins, such that $S$ may not be a prediction set of $f^{-1}\left(\left\{\eta_{2}\right\}\right)$ if $\eta_{2}$ is slightly perturbed. Since the step from $\eta_{2}$ to $\eta_{3}$ crosses a caustic, the number of solutions of $f(z)=\eta_{2}$ and $f(z)=\eta_{3}$ differ. According to Theorem 3.6 $S=f^{-1}\left(\left\{\eta_{2}\right\}\right) \cup\left\{z_{ \pm}\right\}$with the points $z_{ \pm}$from (3.6) is a prediction set of $f^{-1}\left(\left\{\eta_{3}\right\}\right)$, shown in the right plot. 

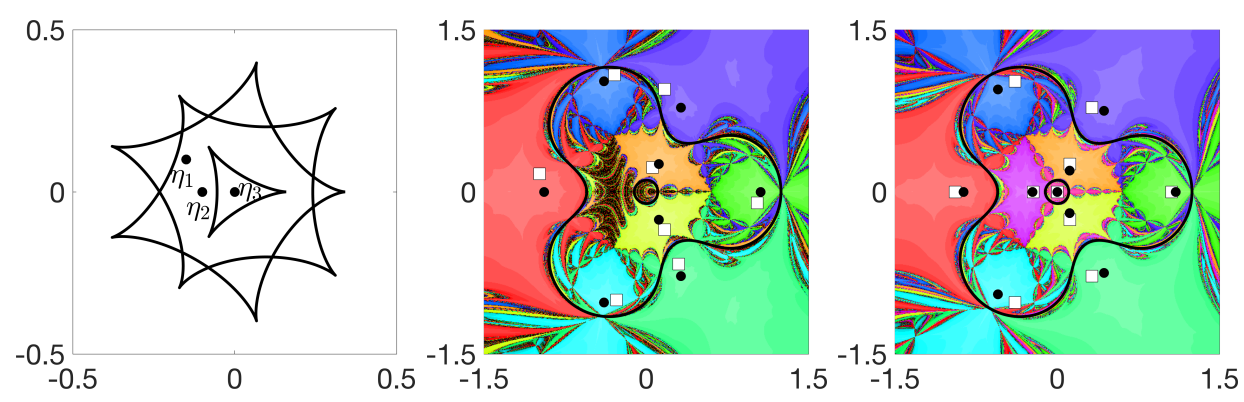

Figure 4: Illustration of Theorems 3.4 and 3.6 for $f(z)=z-\overline{z^{2} /\left(z^{3}-0.6^{3}\right)}$ (see $(5.2)$ below). Left: caustics and $\eta_{1}, \eta_{2}, \eta_{3}$. Middle: prediction set $S=f^{-1}\left(\left\{\eta_{1}\right\}\right)$ (white squares) of $f^{-1}\left(\left\{\eta_{2}\right\}\right)$ (black dots). Right: prediction set $S=f^{-1}\left(\left\{\eta_{2}\right\}\right) \cup\left\{z_{ \pm}\right\}$(white squares) of $f^{-1}\left(\left\{\eta_{3}\right\}\right)$ (black dots).

Theorems 3.2, 3.4 and 3.6 establish the transport of images method if the points $\eta_{k}$ are chosen properly. This motivates the next definition.

Definition 3.9. Let $f$ be a non-degenerate harmonic mapping on $\widehat{\mathbb{C}}$. We call $\left(\eta_{1}, \ldots, \eta_{n}\right) \in \mathbb{C}^{n}$ a transport path from $\eta_{1}$ to $\eta_{n}$ if for each $k=1, \ldots, n-1$ either $f^{-1}\left(\left\{\eta_{k}\right\}\right)$ or $S$ in (3.7) is a prediction set of $f^{-1}\left(\left\{\eta_{k+1}\right\}\right)$.

Along a transport path, all solutions of $f(z)=\eta_{k+1}$ can be computed from the solutions of $f(z)=\eta_{k}$. Next we show the existence of such paths. Here we call $\eta$ a multiple caustic point if $\left|f^{-1}(\{\eta\}) \cap \mathcal{C}\right|>1$.

Theorem 3.10. Let $f$ be a non-degenerate harmonic mapping on $\widehat{\mathbb{C}}$ without non-isolated multiple caustic points, and let $\eta_{s}, \eta_{e} \in \mathbb{C} \backslash f(\mathcal{C})$. Then there exists a transport path from $\eta_{s}$ to $\eta_{e}$.

Proof. We show that there exists a polygonal path from $\eta_{s}$ to $\eta_{e}$, which intersects the caustics only at a finite number of simple fold points. Then this path is refined if necessary.

Let $R$ be the complex plane $\mathbb{C}$ without caustic points that are not simple fold points, which is $\mathbb{C}$ minus a set of isolated points, and hence a region (open and connected), as we discuss next. Since $f$ is non-degenerate, the functions $\partial_{z} f, \overline{\partial_{\bar{z}} f}$ and $\omega$ are rational, see 2.16 . This has several implications. The sets $\mathcal{M}$ from 2.7 and $f(\mathcal{M})$ are finite. The set $\mathcal{C} \backslash \mathcal{M}$ consists of $\operatorname{deg}(\omega)$ many pre-images of the unit circle under $\omega$. There are at most finitely many critical points $z_{0}$ of $f$ with $\omega^{\prime}\left(z_{0}\right)=0$, hence the number of caustic points where the tangent 2.11 does not exist is finite. The number of caustic points where the tangent exists and is zero is finite by Lemma 2.3 (on each of the $\operatorname{deg}(\omega)$ many pre-images of the unit circle, there are at most finitely many points with $\tau=0$, or the whole arc is mapped onto a single point). By assumption, multiple caustic points are isolated.

Next, we prove the existence of a rectifiable path from $\eta_{s}$ to $\eta_{e}$ in $R$ with only finitely many caustic points (simple folds) on it. Since $\eta_{s}, \eta_{e} \notin f(\mathcal{C})$ 
and since the caustics are compact there exists $\delta>0$ such that $D_{\delta}\left(\eta_{s}\right)$, $D_{\delta}\left(\eta_{e}\right)$ contain no caustic points. We consider the increasing sequence of regions $U_{k} \subseteq R$ whose points are 'at most $k$ caustic crossings distant' from $\eta_{s}$, constructed as follows. Let $U_{0}$ be the component (maximal open and connected set) of $R \backslash f(\mathcal{C})$ with $\eta_{s} \in U_{0}$. Suppose that $U_{k} \subseteq R$ has been constructed. Let $U_{k+1}$ be the component of $R \backslash\left(f(\mathcal{C}) \backslash\left(\partial U_{0} \cup \ldots \cup \partial U_{k}\right)\right)$ with $\eta_{s} \in U_{k+1}$. If $\eta_{e} \in U_{k}$, there exists a rectifiable path from $\eta_{s}$ to $\eta_{e}$ in $R$ that intersects $\partial U_{0}, \ldots, \partial U_{k-1}$, and hence has $k$ caustic crossings. If $\eta_{e} \notin U_{k}$ then the boundary of $U_{k}$ in $R$, which consists of caustic arcs, has length at least $2 \pi \delta$, since then $D_{\delta}\left(\eta_{s}\right) \subseteq U_{k}$ and $D_{\delta}\left(\eta_{e}\right) \subseteq R \backslash U_{k}$. Let $m=\left\lceil\frac{L}{2 \pi \delta}\right\rceil$, where $L<\infty$ is the total length of the caustics (see the discussion below Definition 2.5). Then $\eta_{e} \in U_{m}$, and there exists a rectifiable path from $\eta_{s}$ to $\eta_{e}$ with at most $m$ caustic points.

By manipulations in an arbitrary small neighborhood around this path in $R$ we obtain a polygonal path $P=\left(\eta_{1}, \ldots, \eta_{n}\right)$ with $\eta_{1}=\eta_{s}$ and $\eta_{n}=\eta_{e}$, such that: (1) $\eta_{1}, \ldots, \eta_{n} \in \mathbb{C} \backslash f(\mathcal{C}),(2)$ each line segment $\left[\eta_{k}, \eta_{k+1}\right]$ contains at most one caustic point and (3) if the line segment $\left[\eta_{k}, \eta_{k+1}\right]$ contains a caustic point then $\arg \left(\eta_{k+1}-\eta_{k}\right)=\arg ( \pm c)$, with $c$ as in Theorem 3.5 .

We refine this path to get a transport path. First we consider the line segments $\left[\eta_{k}, \eta_{k+1}\right]$, which contain a caustic point $\eta$, but where $S$ in (3.7) is not a prediction set of $f^{-1}\left(\left\{\eta_{k+1}\right\}\right)$. We add two points from $\left[\eta_{k}, \eta_{k+1}\right] \backslash f(\mathcal{C})$ sufficiently close to $\eta$ such that Theorem 3.6 applies to these points. Denote the refined path for simplicity again by $P=\left(\eta_{1}, \ldots, \eta_{n}\right)$. Next we refine the line segments $E=\left[\eta_{k}, \eta_{k+1}\right]$ without caustic points. For all $\eta \in E$ there exists an $\varepsilon(\eta)>0$ such that $f^{-1}(\{\eta\})$ is a prediction set of $f^{-1}(\{\xi\})$ for all $\xi \in D_{\varepsilon(\eta)}(\eta)$ by Theorem 3.4. The family $\left(D_{\varepsilon(\eta)}(\eta)\right)_{\eta \in E}$ is an open covering of the compact set $E$. Hence, there exists a finite subcovering $\left(D_{\varepsilon\left(\kappa_{j}\right)}\left(\kappa_{j}\right)\right)_{j=1, \ldots, \ell}$ with $\kappa_{1}=\eta_{k}$ and $\kappa_{\ell}=\eta_{k+1}$, which gives a partition of $E$, where $f^{-1}\left(\left\{\kappa_{j}\right\}\right)$ is a prediction set of $f^{-1}\left(\left\{\kappa_{j+1}\right\}\right)$ for $j=1, \ldots, \ell-1$. Refining all line segments without caustic points yields a transport path.

Note that non-degenerate harmonic mappings can actually have nonisolated multiple caustic points.

Example 3.11. The harmonic mapping $f(z)=\frac{1}{2}\left(z^{2}-1\right)^{2}+\overline{z^{2}-1}$ from [37, Ex. 5.1] is non-degenerate and maps its critical arcs $\gamma_{ \pm}:[-\pi, \pi] \rightarrow \mathcal{C}$, $\gamma_{ \pm}(t)= \pm \sqrt{1+e^{-i t}}$, onto the same caustic arc.

We close this section by noting the correctness of the transport of images method, provided that 0 is not a caustic point of $f$.

Corollary 3.12. Let $f$ be a non-degenerate harmonic mapping on $\widehat{\mathbb{C}}$ without non-isolated multiple caustic points, such that 0 is not a caustic point. Then there exists a point $\eta_{1} \in \mathbb{C} \backslash f(\mathcal{C})$, such that Theorem 3.2 applies to all poles of $f$, and there exists a transport path from $\eta_{1}$ to 0 . 
Proof. Theorem 3.2 applies to all poles of $f$ for all $\eta_{1} \in \mathbb{C}$ with large enough $\left|\eta_{1}\right|$. Since $f(\mathcal{C})$ is compact, $\eta_{1}$ can be chosen in $\mathbb{C} \backslash f(\mathcal{C})$. Then there exists a transport path from $\eta_{1}$ to 0 by Theorem 3.10 .

Remark 3.13. In Corollary 3.12, the point 0 is not a caustic point of $f$. If 0 is a caustic point then $f$ has (at least) one singular zero $z_{0} \in \mathcal{C}$. Since continuation may run into numerical trouble if solutions are almost singular, special strategies are used. These are usually referred to as the endgame. For systems of analytic functions, two commonly used endgame strategies are the Cauchy endgame [27] and the power series endgame [28]. The setting for the transport of images method is somewhat different since we require the critical set $\mathcal{C}$ in the method. Therefore, we can (numerically) compute the zeros of $f$ in $\mathcal{C}$, which are exactly the singular zeros. Thus, in the endgame we would only have to determine which homotopy curves intersect at $z_{0}$. This can be done with (2) and (3a) in Section 3.4 if $f$ is non-degenerate and $\omega^{\prime}\left(z_{0}\right) \neq 0$.

\subsection{Analysis of the homotopy curves}

We now consider the continuous problem behind our (discrete) computation and describe the homotopy curves, i.e., how the solution set of $f(z)=\eta(t)$ varies with $t$, where $f: \Omega \rightarrow \mathbb{C}$ is a harmonic mapping and $\eta:[a, b] \rightarrow \mathbb{C}$, $t \mapsto \eta(t)$, is a (continuous) path. We analyze the solution set locally with results from [23, 36, 37]. Combining the local results gives the global picture. We distinguish the cases (1) $z_{0} \in \Omega \backslash \mathcal{C},(2) z_{0} \in \mathcal{M}$ and (3) $z_{0} \in \mathcal{C} \backslash \mathcal{M}$.

(1) Let $z_{0} \in \Omega \backslash \mathcal{C}$, i.e., the Jacobian of $f$ is non-zero at $z_{0}$. By the inverse function theorem there exists an $\varepsilon>0$ and an open set $V \subseteq \mathbb{C}$ such that $f: D_{\varepsilon}\left(z_{0}\right) \rightarrow V$ is a diffeomorphism. Thus, a curve $\eta(t)$ in $V$ has a unique pre-image curve $z(t)=f^{-1}(\eta(t))$. In particular, the homotopy curves can intersect only at critical points.

(2) Let $z_{0} \in \mathcal{M}$ from (2.7). Then $f$ has the form

$$
f(z)=a_{0}+\sum_{k=n}^{\infty} a_{k}\left(z-z_{0}\right)^{k}+\overline{b_{0}+\sum_{k=n}^{\infty} b_{k}\left(z-z_{0}\right)^{k}}
$$

with $n \geq 2$ and $\left|a_{n}\right| \neq\left|b_{n}\right|$. If $\eta(t)$ passes through $f\left(z_{0}\right)$ then exactly $n$ homotopy curves intersect at $z_{0}$ with equispaced angles, since $f(z)=\eta(t)$ is locally to leading order $f\left(z_{0}\right)+a_{n}\left(z-z_{0}\right)^{n}+\overline{b_{n}\left(z-z_{0}\right)^{n}}=\eta(t)$. The latter can be uniquely solved for $\left(z-z_{0}\right)^{n}$ by

$$
\left(z-z_{0}\right)^{n}=\frac{\bar{a}_{n}\left(\eta(t)-f\left(z_{0}\right)\right)-\overline{b_{n}\left(\eta(t)-f\left(z_{0}\right)\right)}}{\left|a_{n}\right|^{2}-\left|b_{n}\right|^{2}},
$$

see [36, Lem. 4.2], which yields the $n$ solutions. 

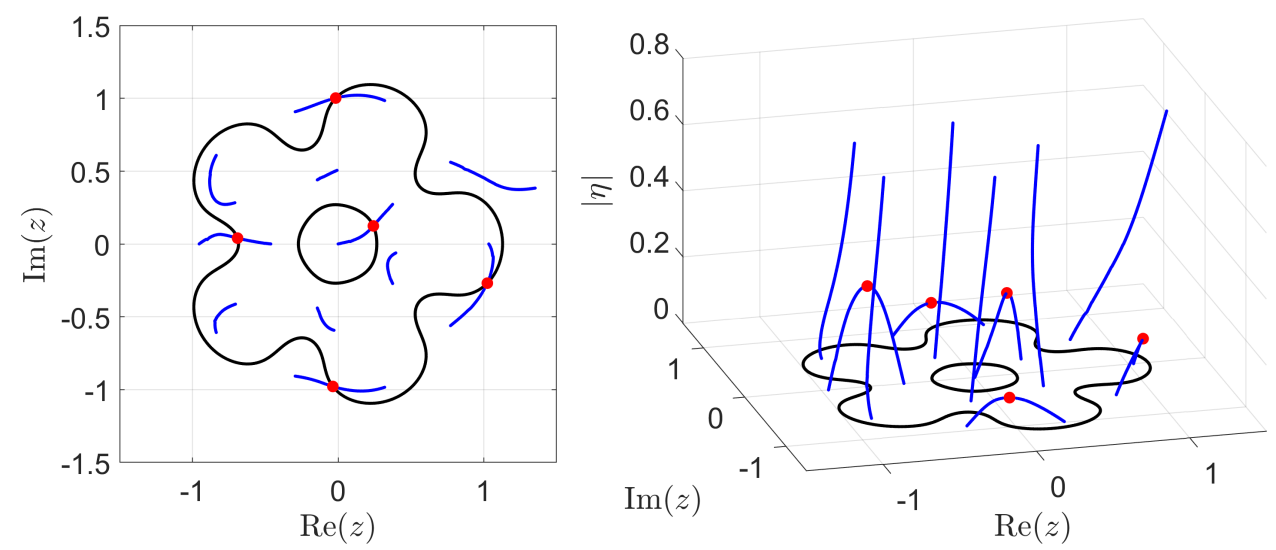

Figure 5: Computed homotopy curves (blue) with turning points (red dots) in Example 3.14 and critical set of $f$ (black).

(3) For $z_{0} \in \mathcal{C} \backslash \mathcal{M}$ the situation is more involved. By [23, Thm. 2.1], a harmonic mapping is either (a) light, (b) has zero Jacobian or (c) is constant on some arc of $\mathcal{C} \backslash \mathcal{M}$. We discuss these three cases.

(3a) Let $f$ be a light harmonic mapping. Essentially, the behavior of the homotopy curves at $z_{0}$ depends on the tangent to the caustics at $f\left(z_{0}\right)$ and hence on $\psi$ from $(2.12)$. Let $z_{0} \in \mathcal{C} \backslash \mathcal{M}$. Then $z_{0}$ is a zero of order $\ell \geq 0$ of $\partial_{z} f$ (and of $\left.\overline{\partial_{\bar{z}} f}\right)$. First let $\omega^{\prime}\left(z_{0}\right) \neq 0$, so that $z_{0}=\gamma\left(t_{0}\right)$ is a point on a critical curve that is not a self-intersection point of the critical curves.

1. If $\psi\left(t_{0}\right)$ is non-zero $\left(f\left(z_{0}\right)\right.$ is a fold caustic point and consequently $\ell=0)$ then $z_{0}$ is a turning point of the homotopy curves, i.e., two curves start or end at $z_{0}$ if $\eta(t)$ crosses the caustics at $f\left(z_{0}\right)$. This follows from Theorem 3.5 or [23, Thm. 5.1] and is illustrated in [37, Fig. 5].

2. More generally, if $\psi$ does not change sign at $t_{0}$ then [23, Thm. 5.1] implies that $\ell$ homotopy curves intersect at $z_{0}$ and two homotopy curves start or end at $z_{0}$, if $\eta(t)$ crosses the caustics at $f\left(z_{0}\right)$. Hence, $z_{0}$ is a turning point and, if $\ell>0$, a bifurcation point of the homotopy curves.

3. If $\psi$ changes sign at $t_{0}$ (i.e., $f\left(z_{0}\right)$ is a cusp) then by [23, Thm. 5.1] $z_{0}$ is a bifurcation point where $\ell+1$ homotopy curves intersect and two homotopy curves start or end, if $\eta(t)$ crosses the caustics at $f\left(z_{0}\right)$.

For $\omega^{\prime}\left(z_{0}\right)=0$ there is only an upper bound on the local valence of $f$ at $z_{0}$ in [23, Sect. 6] and hence on the number of homotopy curves, which also depends on the caustic tiles bordering $f\left(z_{0}\right)$ that are traversed by $\eta(t)$.

Example 3.14. Consider the harmonic mapping (5.2) with $n=5$ and $r=0.6$, which is light and non-degenerate. Figure 5 shows the computed homotopy curves where $\eta=\eta(t)$ parametrizes the segment from 
$0.6699+0.1795 i$ to 0 , which intersects the caustics of $f$ only at simple fold points. Along this path each caustic crossing produces two additional solutions. The corresponding turning points of the homotopy curves are marked with red dots. Figure 5 shows the solutions $z(t)$ of $f(z)=\eta(t)$ in the $z$-plane (left) and over the $z$-plane at height $|\eta(t)|$ (right).

(3b) Let $f$ be a harmonic mapping with $J_{f} \equiv 0$, i.e., $\mathcal{C}=\Omega$. Then $f$ has the form $f(z)=a \operatorname{Re}(h(z))+b$ with $a, b \in \mathbb{C}, a \neq 0$, and an analytic function $h$; see [23, Lem. 2.1] or [30, Lem. 4.7]. (If $\Omega$ is not simply connected, $h$ may be multi-valued, but $\operatorname{Re}(h)$ is single-valued.) Then $f(\Omega) \subseteq\{a t+b: t \in \mathbb{R}\}$, and $f(z)=\eta$ if and only if $\operatorname{Re}(h(z))=(\eta-b) / a$. Since $h$ is analytic, it is either constant or has the open mapping property, which shows that the solution set of $f(z)=\eta$ is either empty, $\Omega$ or a union of curves. Therefore, along a path $\eta(t)$ crossing the caustics of $f$, the solutions do not form curves depending on $t$.

Example 3.15. The Jacobian of $f(z)=z+\bar{z}$ vanishes identically. Clearly, $f(z)=\eta \in \mathbb{C} \backslash \mathbb{R}$ has no solution, while $f(z)=\eta \in \mathbb{R}$ is solved by all $z$ with $\operatorname{Re}(z)=\eta / 2$.

(3c) Let $f$ be a harmonic mapping that is constant on some arc $\Gamma$ of $\mathcal{C} \backslash \mathcal{M}$, and $z_{0} \in \Gamma$. Then $f$ maps $\Gamma$ onto the single point $f\left(z_{0}\right)$. For $\eta(t)$ not equal to $f\left(z_{0}\right)$ (or any similar point), the homotopy curves are as for light harmonic mappings. However, if $\eta(t)=f\left(z_{0}\right)$ then the solution set also contains all points of $\Gamma$, and the solutions do not form curves depending on $t$.

Example 3.16. The non-degenerate harmonic mapping $f(z)=z-1 / \bar{z}$ is not light since $f^{-1}(\{0\})=\{z \in \mathbb{C}:|z|=1\}=\mathcal{C}$. Let $\eta \in \mathbb{C} \backslash\{0\}$. Then $f(z)=\eta$ has the two solutions

$$
z_{ \pm}(\eta)=\frac{1 \pm \sqrt{1+4|\eta|^{-2}}}{2} \eta
$$

which satisfy $\left|z_{+}(\eta)\right|>1$ and $\left|z_{-}(\eta)\right|<1$, and $\left|z_{ \pm}(\eta)\right| \rightarrow 1$ if $\eta \rightarrow 0$. Figure 6 shows a path $\eta(t)$ crossing the origin (panel 1) and the corresponding homotopy curves (panel 2). For $\eta(t)=0$ the two solutions are replaced by all points on the unit circle. Panels 3 and 4 show a similar example with a non-smooth path $\eta(t)$, which leads to homotopy curves exiting $\mathcal{C}$ at different points.

The analysis of the homotopy curves also confirms that Definition 3.9 of a transport path is reasonable. The points $\eta_{k}$ are in $\mathbb{C} \backslash f(\mathcal{C})$ to avoid a zero Jacobian and potentially infinitely many solutions. Moreover, to avoid bifurcation points of the homotopy curves, transport paths cross the caustics only at fold points, which can be handled by Theorem 3.5 

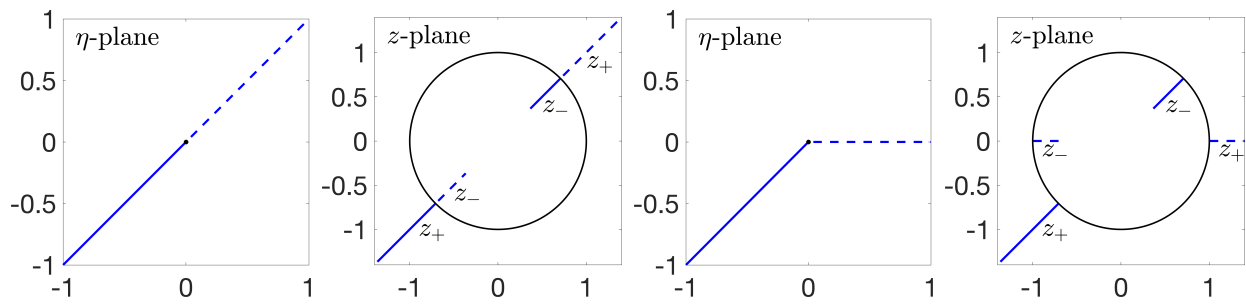

Figure 6: Homotopy curves of $f(z)=z-1 / \bar{z}$ for two paths $\eta(t)$ (panels 1 and 3 ), divided in a solid and dashed blue part, and corresponding pre-images (panels 2 and 4); see Example 3.16. The black circle is $\mathcal{C}=f^{-1}(\{0\})$.

\section{Implementation}

A MATLAB implementation of our method is freely available at

$$
\text { https://github.com/transportofimages/ }
$$

This toolbox contains routines to compute the zeros of non-degenerate harmonic mappings and the critical curves and caustics. Moreover, it contains $\mathrm{m}$-files to reproduce all examples in Section 5 . We briefly describe two key aspects of our implementation.

\subsection{Computation of the caustics}

The transport of images methods bases essentially upon the correct handling of caustic crossings. To compute the caustics we first compute the critical curves. Critical points $z \in \mathcal{C} \backslash \mathcal{M}$ satisfy $\omega(z)=e^{i t}$ with $t \in[0,2 \pi[$; see $(2.9)$. Since $\omega$ is rational for non-degenerate harmonic mappings, $\omega(z)=e^{i t}$ is equivalent to a polynomial equation with $\operatorname{deg}(\omega)$ many solutions. We first solve $\omega(z)=1$ via the polynomial equation. Then, for $0=t_{1}<t_{2}<\ldots<$ $t_{k}<2 \pi$ we successively solve $\omega(z)=e^{i t_{j+1}}$ with Newton's method (with initial points $\left.\omega^{-1}\left(\left\{e^{i t_{j}}\right\}\right)\right)$. If successful, this procedure gives $\operatorname{deg}(\omega)$ many (discretized) critical arcs, parametrized according to 2.10). Gluing them together yields the critical curves. Finally, the image under $f$ of the critical curves is a discretization of the caustics of $f$.

\subsection{Construction of transport paths}

We construct a transport path to 0 along $R_{\theta}=\left\{r e^{i \theta}: r>0\right\}, \theta \in[0,2 \pi[$. Beneficially, the intersection points of the caustic and the ray $R_{\theta}$ can be read off from the argument of the caustic points. Also, as discussed in Remark 3.7, crossing the caustics in a specific direction is not essential, as long as the crossing direction is not tangential to the caustics. For brevity we still call $\left(\eta_{1}, \ldots, \eta_{n}\right) \in \mathbb{C}^{n}$ a transport path if $f^{-1}\left(\left\{\eta_{k}\right\}\right)$ or $S$ in $(3.7)$ is a prediction set of $f^{-1}\left(\left\{\eta_{k+1}\right\}\right)$, even if the step from $\eta_{k}$ to $\eta_{k+1}$ is not in the direction $c$ in 
Theorem 3.6, i.e., even if $\arg \left(\eta_{k+1}-\eta_{k}\right) \neq \arg ( \pm c)$. Determining a suitable angle $\theta \in\left[0,2 \pi\right.$ [ for $R_{\theta}$ can be challenging. In particular, the transport phase (along $R_{\theta}$ ) may fail if too small step sizes are required, or if $R_{\theta}$ is tangential or almost tangential to the caustics, or if $R_{\theta}$ intersects the caustic at or near cusps or other non-fold points. To overcome this difficulty we choose the angle $\theta \in[0,2 \pi[$ at random (e.g., uniformly distributed). If the transport phase with $\theta$ is not successful we restart with a new random angle.

We construct a transport path to 0 along $R_{\theta}$ as follows:

(1) Set $\eta_{1}=2 e^{i \theta} \max _{z \in \mathcal{C}}|f(z)|$. If Theorem 3.2 with $\eta_{1}$ does not apply to all poles of $f$, e.g., two distinct points $\zeta_{j}$ and $\zeta_{\ell}$ are attracted by the same solution of $f(z)=\eta_{1}$, we increase $\left|\eta_{1}\right|$ and proceed. Eventually, $\left|\eta_{1}\right|$ is large enough and Theorem 3.2 applies to all poles of $f$ simultaneously.

(2) Let $\left\{\xi_{1}, \ldots, \xi_{\ell}\right\}=f(\mathcal{C}) \cap R_{\theta}$ with $\left|\xi_{1}\right|>\ldots>\left|\xi_{\ell}\right|>0$. We compute these intersection points from the discretized caustics with bisection. For each $\xi_{k}$ let $\eta_{2 k}, \eta_{2 k+1} \in R_{\theta}$ with equal distance to $\xi_{k}$, and such that $\left|\eta_{1}\right|>$ $\left|\eta_{2}\right|>\ldots>\left|\eta_{2 \ell+1}\right|>0$. Then $P=\left(\eta_{1}, \eta_{2}, \ldots, \eta_{2 \ell+1}, 0\right)$ is a potential transport path.

(3) We refine $P$ recursively with the following divide-and-conquer scheme to obtain a transport path.

(3a) If the step from $\eta_{j}$ to $\eta_{j+1}$, where the line segment $\left[\eta_{j}, \eta_{j+1}\right]$ does not intersect the caustics, is unsuccessful, i.e., if $f^{-1}\left(\left\{\eta_{j}\right\}\right)$ is not a prediction set of $f^{-1}\left(\left\{\eta_{j+1}\right\}\right)$, we divide the step from $\eta_{j}$ to $\eta_{j+1}$ into two substeps by inserting $\eta^{\prime}=\left(\eta_{j}+\eta_{j+1}\right) / 2$ into $P$.

(3b) If the step from $\eta_{j}$ to $\eta_{j+1}$, where the line segment $\left[\eta_{j}, \eta_{j+1}\right]$ intersects the caustic, is unsuccessful, i.e., if $S$ in (3.7) is not a prediction set of $f^{-1}\left(\left\{\eta_{j+1}\right\}\right)$, we divide the step from $\eta_{j}$ to $\eta_{j+1}$ into three substeps by inserting $\eta_{1}^{\prime}=\left(3 \eta_{j}+\eta_{j+1}\right) / 4$ and $\eta_{2}^{\prime}=\left(\eta_{j}+3 \eta_{j+1}\right) / 4$ into $P$.

(4) We proceed, dividing the steps in (3a) and (3b) until $P$ is a transport path, or until we reached a prescribed recursion depth. In the latter case we restart with a new random angle $\theta$ in (1).

\section{$5 \quad$ Numerical examples}

In order to verify the numerical results we test our method on the following functions, for which the number of zeros is known analytically.

1. Wilmshurst's harmonic polynomial

$$
f(z)=(z-1)^{n}+z^{n}+\overline{i(z-1)^{n}-i z^{n}}, \quad n \geq 1,
$$

is non-degenerate and has exactly $n^{2}$ zeros. This is the maximum number of zeros of a harmonic polynomial $f(z)=p(z)+\overline{q(z)}$ with $\operatorname{deg}(p)=n$ and $\operatorname{deg}(q)<n$; see [44]. 

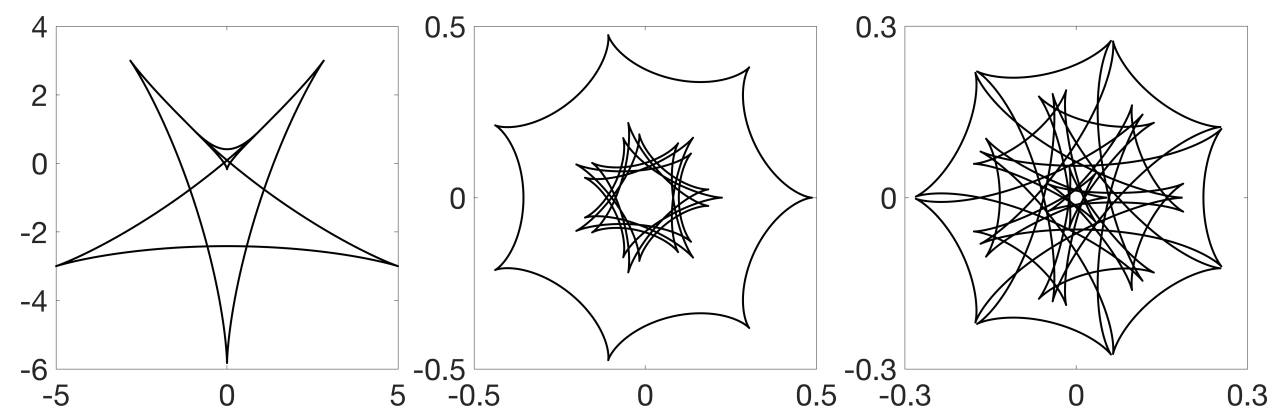

Figure 7: Caustics of harmonic mappings: (5.1) with $n=3$ (left), (5.2) with $n=7$ and $\rho=0.7$ (middle), (5.3) with $n=7, \rho=0.7$ and $\varepsilon=0.1$ (right).

2. The rational harmonic mapping in Mao, Petters and Witt [24],

$$
f(z)=z-\overline{\left(\frac{z^{n-1}}{z^{n}-\rho^{n}}\right)}, \quad n \geq 3, \quad \rho>0,
$$

is non-degenerate and has exactly $3 n+1$ zeros for $\rho<\rho_{c}, 2 n+1$ zeros for $\rho=\rho_{c}$ and $n+1$ zeros for $\rho>\rho_{c}$, where

$$
\rho_{c}=\left(\frac{n-2}{n}\right)^{\frac{1}{2}}\left(\frac{2}{n-2}\right)^{\frac{1}{n}} ;
$$

see [21, Prop. 2.1].

3. Rhie's rational harmonic mapping

$$
f(z)=z-\overline{\left((1-\varepsilon) \frac{z^{n-1}}{z^{n}-\rho^{n}}+\frac{\varepsilon}{z}\right)}, \quad n \geq 3, \quad \rho, \varepsilon>0,
$$

is non-degenerate and has exactly $5 n$ zeros if $\rho<\rho_{c}$ (as above) and if $\varepsilon<\varepsilon^{*}$, where $\varepsilon^{*}$ depends on $n$ and $\rho$; see [21, Thm. 2.2] for details. This is the maximum number of zeros of a harmonic mapping $f(z)=z-\overline{r(z)}$ with a rational function $r$ of degree $n+1 \geq 2$; see [14, Thm. 1].

These functions should be challenging for the transport of images method since they have a large number of zeros and very nested caustics; see Figure 7 . In gravitational lensing, the functions (5.2), 5.3 and their zeros are of interest; see [15, 21].

The following computations have been performed in MATLAB R2019b on an i7-7700 $4 \times 3.60 \mathrm{GHz}$ CPU with 16 GB RAM using our implementation ${ }^{3}$, which also contains $\mathrm{m}$-files to reproduce all examples.

First, we compute the zeros of (5.1), (5.2), (5.3), and of the transcendental harmonic mapping (2.19) with the transport of images method. Here

\footnotetext{
$3_{\text {https://github.com/transportofimages/ }}^{3}$
} 


\begin{tabular}{|lrrrrrr|}
\hline Function & Zeros & Max. res. & Time & N. iter. & Steps & Ref. \\
\hline$(2.19$ & 4 & $6.6613 \mathrm{e}-16$ & $36 \mathrm{~ms}$ & 237 & 8 & 2 \\
$(5.1)$ & 9 & $1.7764 \mathrm{e}-15$ & $44 \mathrm{~ms}$ & 291 & 8 & 1 \\
$(\overline{5.2}$ & 22 & $8.9509 \mathrm{e}-16$ & $62 \mathrm{~ms}$ & 1646 & 19 & 4 \\
$(5.3)$ & 35 & $7.8505 \mathrm{e}-16$ & $85 \mathrm{~ms}$ & 3851 & 40 & 9 \\
\hline
\end{tabular}

Table 1: The transport of images method for (2.19), (5.1) with $n=3,(5.2)$ with $n=7, \rho=0.7$, and (5.3) with $n=7, \rho=0.7, \varepsilon=0.1$ : maximal residual (max. res.), computation time, number of harmonic Newton iterations (N. iter.), number of transport steps, and number of step refinements (Ref.).

we fix the angle $\theta=\pi / 50$ in the construction of the transport path (see Section 4.2). The results are displayed in Table 1. We make the following three main observations: (1) The transport of images method finishes with the correct number of zeros for each function, in particular for the transcendental harmonic mapping (2.19). The logarithmic term makes a symbolic computation of the zeros difficult, e.g., Mathematica cannot determine the zeros of this function. We emphasize that the number of zeros is not an input parameter of our method. (2) The results are computed within a fraction of a second on a standard computer. (3) In each case the computed results are very accurate, as shown by the residual $\left|f\left(z_{j}\right)\right|$ at the computed zeros, which is in the order of the machine precision. Table 1 shows the maximal residual over all computed zeros for the respective functions. Furthermore, we see that a step refinement as described in Section 4.2 was necessary for all functions. The number of (harmonic) Newton iterations also includes iterations that are necessary to decide whether a step has to be refined.

Figure 8 illustrates the transport of images method for (5.1) with $n=3$. Panels 1 and 2 display the transport path and a zoom-in close to the origin. The remaining panels show the dynamics of the harmonic Newton maps $H_{f-\eta_{k}}$ for $k=1,2, \ldots, 9$. Recall from Remark 3.8 that points which are attracted by the same solution have the same color. The initial phase is visualized in the top right plot. Every solution of $f(z)=\eta_{1}$ (black dots) attracts exactly one of the initial points (white squares), constructed as in Section 3.2, hence these initial points form a prediction set of $f^{-1}\left(\left\{\eta_{1}\right\}\right)$. For aesthetic reasons we have set $\eta_{1}=6 e^{i \pi / 50}$ by hand. The remaining panels visualize the steps in the transport phase, again with the solutions of $f(z)=$ $\eta_{k}$ (black dots) and the prediction sets (white squares). In particular, we see how the basins of attraction evolve while $\eta_{k}$ 'travels' along the transport path. New basins and their respective solutions appear in pairs for $\eta_{3}$ (green and cyan), $\eta_{5}$ (blue and light blue) and $\eta_{7}$ (pink and purple), right after a caustic crossing, as predicted by the theory. For each step the prediction set of $f^{-1}\left(\left\{\eta_{k+1}\right\}\right)$ consists of $f^{-1}\left(\left\{\eta_{k}\right\}\right)$ (black dots in the previous panel), and of the points $z_{ \pm}$from Theorem 3.5 in case of a caustic crossing. We 

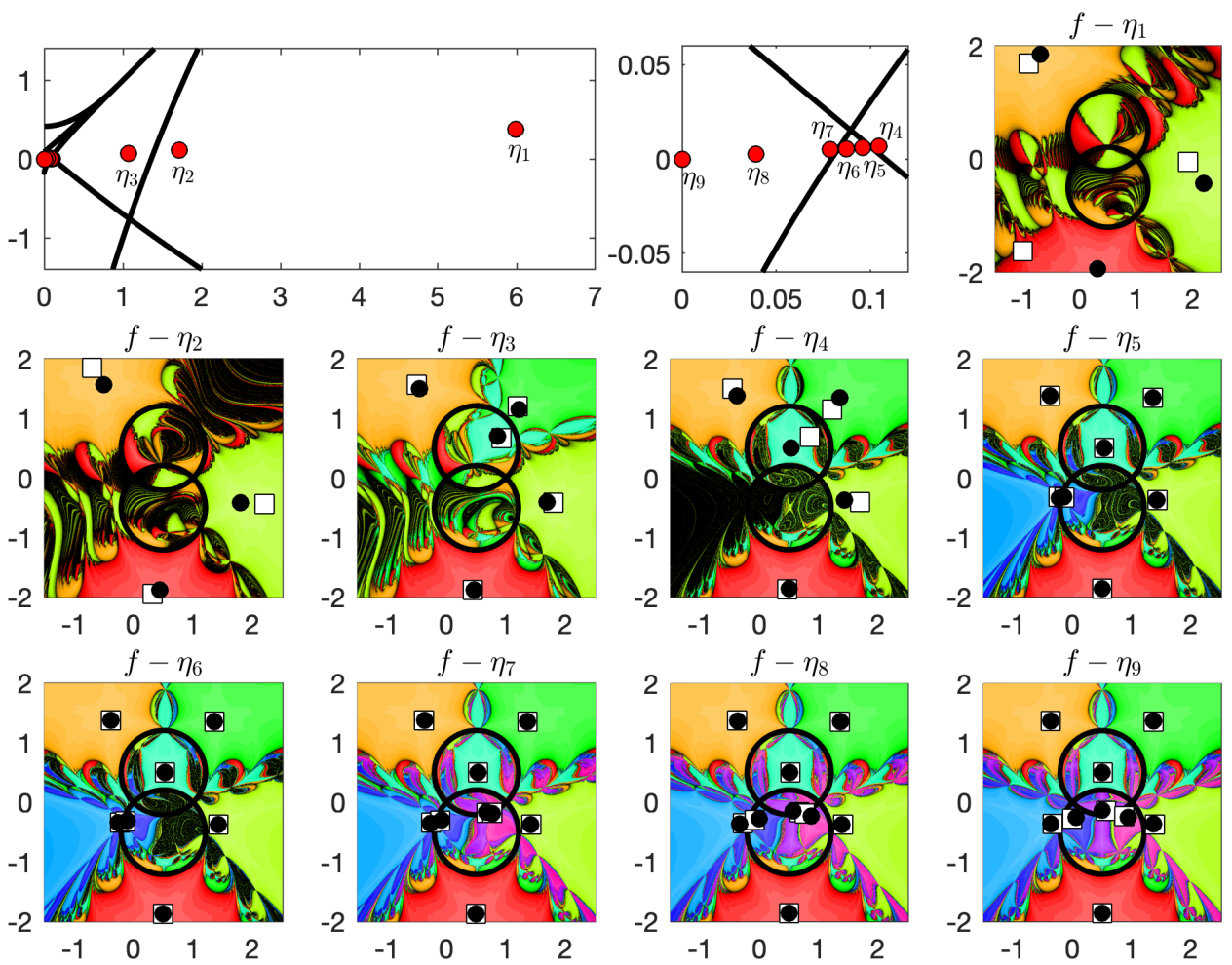

Figure 8: Transport of images method for (5.1) with $n=3$ : Transport path and dynamics of $H_{f-\eta_{k}}$ with prediction set (white squares) and zeros of $f-\eta_{k}$ (black dots).

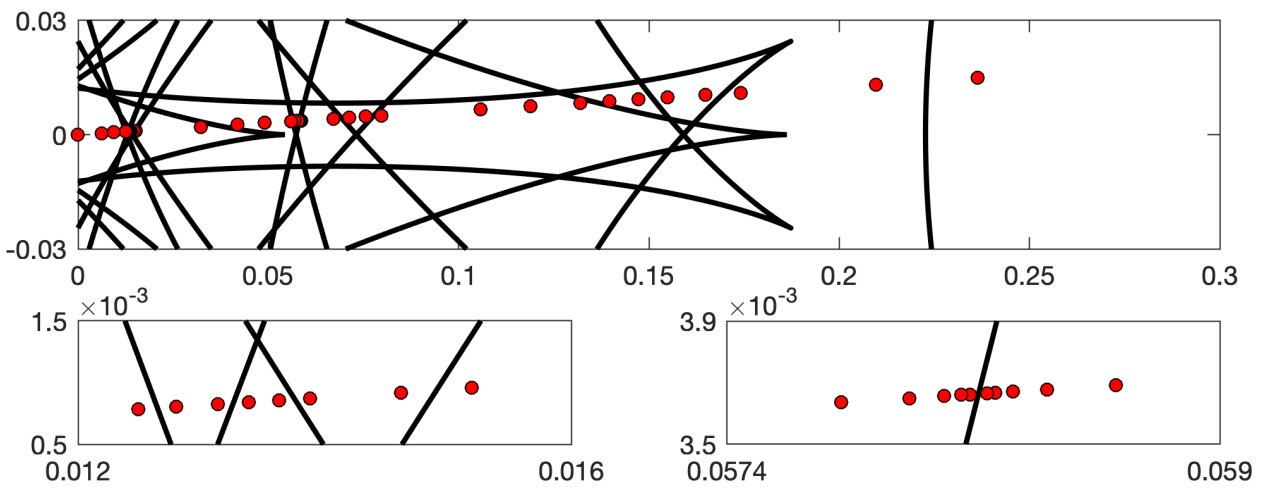

Figure 9: Transport path for (5.3) with $n=7, \rho=0.7, \varepsilon=0.1$ and $\theta=\frac{\pi}{50}$.

see that the 'new' solutions are close to the predicted points $z_{ \pm}$. Note that Theorem 3.4 does not apply to the step from $\eta_{7}$ to $\eta_{9}$. Therefore, this step was divided by introducing the point $\eta_{8}=\left(\eta_{7}+\eta_{9}\right) / 2$. A transport path with more step refinements, but for the function (5.3), is displayed in Figure 9 .

Next, we compute the zeros of the function (5.2) for different values 


\begin{tabular}{|rrrrr|}
\hline$n$ & Zer. & N. iter. & Rest. & Time \\
\hline 3 & 10 & 543.98 & 0.00 & $37 \mathrm{~ms}$ \\
4 & 13 & 926.68 & 0.00 & $45 \mathrm{~ms}$ \\
5 & 16 & 1328.06 & 0.02 & $50 \mathrm{~ms}$ \\
6 & 19 & 1432.50 & 0.00 & $53 \mathrm{~ms}$ \\
7 & 22 & 1765.74 & 0.02 & $60 \mathrm{~ms}$ \\
8 & 25 & 2253.86 & 0.02 & $68 \mathrm{~ms}$ \\
9 & 28 & 2653.40 & 0.00 & $73 \mathrm{~ms}$ \\
10 & 31 & 3126.28 & 0.04 & $81 \mathrm{~ms}$ \\
11 & 34 & 3393.04 & 0.00 & $88 \mathrm{~ms}$ \\
12 & 37 & 3926.20 & 0.02 & $97 \mathrm{~ms}$ \\
3
\end{tabular} \mid \begin{tabular}{rrrrr|}
4 & 15 & 948.46 & 0.00 & $44 \mathrm{~ms}$ \\
5 & 25 & 2046.24 & 0.02 & $61 \mathrm{~ms}$ \\
6 & 30 & 3014.40 & 0.00 & $70 \mathrm{~ms}$ \\
7 & 35 & 3590.90 & 0.04 & $81 \mathrm{~ms}$ \\
8 & 40 & 4989.04 & 0.00 & $93 \mathrm{~ms}$ \\
9 & 45 & 5820.60 & 0.06 & $107 \mathrm{~ms}$ \\
10 & 50 & 6941.06 & 0.06 & $120 \mathrm{~ms}$ \\
11 & 55 & 8035.50 & 0.10 & $137 \mathrm{~ms}$ \\
12 & 60 & 9154.42 & 0.04 & $147 \mathrm{~ms}$ \\
\hline
\end{tabular}

Table 2: The transport of images method averaged over 50 instances. Left: 5.2 for $\rho \in\left[0.7, \rho_{c}[\right.$. Right: 5.3$]$ for $\rho \in\left[0.7, \rho_{c}\left[\right.\right.$ and $\varepsilon \in\left[\frac{\varepsilon^{*}}{2}, \frac{3 \varepsilon^{*}}{4}\right]$. Number of harmonic Newton iterations (N.iter.), number of restarts with new angle $\theta$ (Rest.), computation time.

\begin{tabular}{|lcccc|}
\hline Function & Zeros & Max. res. & Restarts & Time \\
\hline 5.2 & 301 & $6.4737 \mathrm{e}-16$ & 0 & $5.36 \mathrm{~s}$ \\
$(5.3)$ & 125 & $1.3552 \mathrm{e}-15$ & 7 & $2.52 \mathrm{~s}$ \\
\hline
\end{tabular}

Table 3: The transport of images method for 5.2 with $n=100, \rho=0.94$, and (5.3) with $n=25, \rho=0.9, \varepsilon=0.4$.

of $n$ with the transport of images method (without fixing the angle $\theta$ ). For each $n=3,4, \ldots, 12$ we consider 50 instances of 5.2 with randomized $\rho \in\left[0.7, \rho_{c}[\right.$, and apply our method to these functions. Similarly, we consider 50 instances of the function 5.3 with random $\rho \in\left[0.7, \rho_{c}\left[\right.\right.$ and $\varepsilon \in\left[\frac{\varepsilon^{*}}{2}, \frac{3 \varepsilon^{*}}{4}\right]$. The quantities $\rho$ and $\varepsilon$ are uniformly distributed in the respective intervals. Table 2 shows the computed number of zeros, the number of harmonic Newton iterations, the number of restarts with a new angle $\theta$ and the computation time. For each $n$, the quantities are averaged over all instances. We observe the following. First of all, the transport of images method terminates with the correct number of zeros for all $n$ and all instances. For most instances the transport along the first ray is already successful. For the remaining ones a restart with a new angle is necessary to compute the zeros. In this example, computing the caustics takes roughly half of the overall computation time. This can be exploited when solving $f(z)=\eta_{k}$ for several $\eta_{k} \in \mathbb{C}$, which is a scenario in the astrophysical application in [33, Sect. 10.5].

In our next example we consider (5.2) with $n=100$ and $\rho=0.94$, and (5.3) with $n=25, \rho=0.9$ and $\varepsilon=0.4$. Note that every transport path along a ray $R_{\theta}$ intersects the caustics at least 100 times for the first function and at least 50 times for the second function. The transport of images method computes all zeros in a few seconds on a standard computer; see 

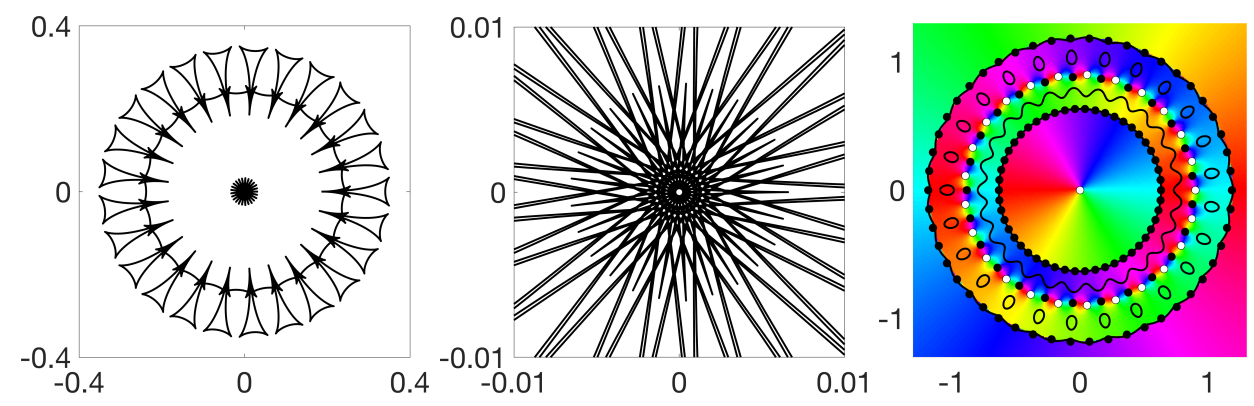

Figure 10: The harmonic mapping $f(z)=z-\overline{\left(0.6 \frac{z^{24}}{z^{25}-0.9^{25}}+0.4 \frac{1}{z}\right)}$; see (5.3). Left: caustics. Middle: zoom of caustics. Right: phase plot with critical curves, zeros (black dots) and poles (white dots).

Table 3. The residuals are close to the machine precision. In contrast to the examples in Table 2, seven restarts with a new angle $\theta$ are necessary for (5.3) with $n=25$. This is due to the extremely nested caustics; see Figure 10 (left and middle). The 125 zeros and 26 poles of the second function are displayed in a phase plot in Figure 10. In a phase plot of $f$ the complex plane is colored according to the phase $f(z) /|f(z)|$; see [43] for an extensive discussion of phase plots and their applications.

As mentioned in the introduction we are not aware of any specialized software to compute all zeros of harmonic mappings. However, we compare our method with the general purpose root finder from [29], which is based on Bézout resultants. There are two implementations of this method: rootsb ${ }^{4}$ and the roots command of Chebfun2 $2^{5}$. Chebfun2 [42] is the state-of-theart toolbox for numerical computation with (real or complex) smooth and bounded functions on a rectangle in the plane. The idea of [29] is to find the zeros of two (real-valued) smooth functions $f(x, y)=g(x, y)=0$ by first computing polynomial approximants $p(x, y)$ and $q(x, y)$ that are accurate to machine precision in the supremum norm relatively to $f$ and $g$, respectively, and then solving the polynomial system $p(x, y)=q(x, y)=0$. In this procedure the polynomial approximants are locally resampled if appropriate. While rootsb uses the original functions, Chebfun2 uses the computed interpolant for the resampling.

We consider again the functions in Table2. To compute the zeros of 5.2 ) and (5.3) with Chebfun2 and rootsb we multiply them by their respective denominators to remove the poles and thus obtain the functions

$$
\begin{aligned}
& F_{1}(z)=z\left(\bar{z}^{n}-\rho^{n}\right)-\bar{z}^{n-1}, \\
& F_{2}(z)=\left(|z|^{2}-1\right) \bar{z}^{n}+\left(\varepsilon-|z|^{2}\right) \rho^{n} .
\end{aligned}
$$

\footnotetext{
${ }^{4}$ rootsb, version of March 20, 2021, https://de.mathworks.com/matlabcentral/ fileexchange/44084-computing-common-zeros-of-two-bivariate-functions

${ }^{5}$ Chebfun2, version of September 30, 2020, www.chebfun.org.
} 

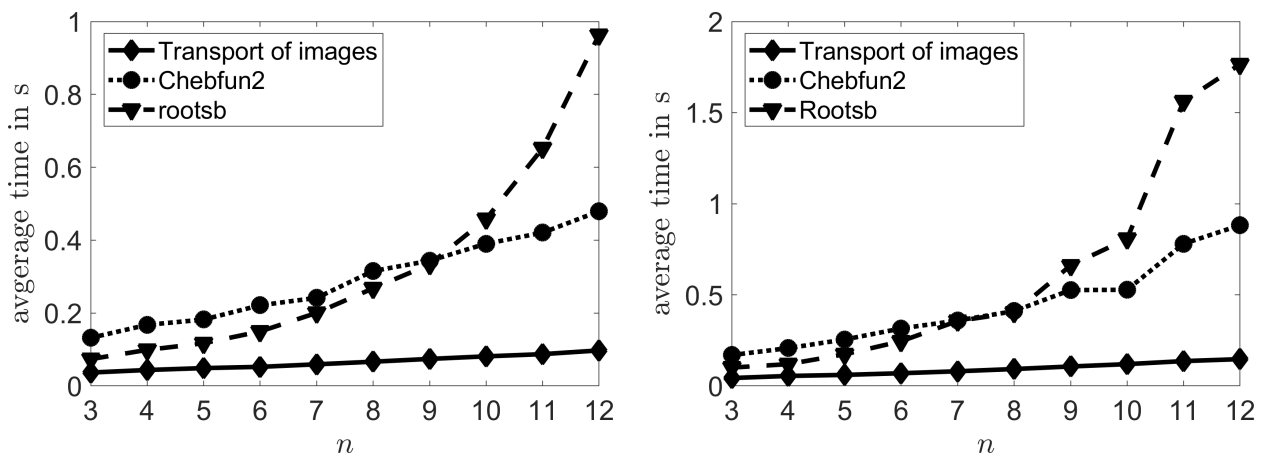

Figure 11: Timings of the transport of images method, Chebfun2 and rootsb averaged over the 50 instances for $(5.2)$ (left) and (5.3) (right) as in Table 2 .

\begin{tabular}{|l|rr|rr|rr|}
\hline Function & \multicolumn{2}{|c|}{ Transport of images } & \multicolumn{2}{|c|}{ Chebfun2 } & \multicolumn{2}{c|}{ rootsb } \\
& Zeros & Time & Zeros & Time & Zeros & Time \\
\hline$(5.2)$ & 301 & $5.36 \mathrm{~s}$ & 10017 & $45.73 \mathrm{~s}$ & 301 & $60.93 \mathrm{~s}$ \\
$(\overline{5.3}$ & 125 & $2.52 \mathrm{~s}$ & 95 & $9.98 \mathrm{~s}$ & 75 & $10.78 \mathrm{~s}$ \\
\hline
\end{tabular}

Table 4: Comparison of the transport of images method with Chebfun2 and rootsb for the functions $(5.2$ with $n=100, \rho=0.94$, and (5.3) with $n=25$, $\rho=0.9, \varepsilon=0.4$, see also Table 3 .

We use the square $R=[-1.2,1.2] \times[-1.2,1.2]$, which contains all zeros of $F_{1}$ and $F_{2}$ for all $n=3,4, \ldots, 12$ and all $\rho$ and $\varepsilon$ as above. Note that the supremum norm of $F_{j}$ on $R$ grows exponentially with $n$. Despite this difficulty, Chebfun2 and rootsb compute all zeros of $F_{1}$ for all instances. For $F_{2}$, rootsb computes all zeros of all instances, and Chebfun2 returns the correct number of zeros for all instances for $n=3,4, \ldots, 9$ and $n=11$, but a wrong number of zeros for 5 out of 50 instances for $n=10$ or $n=12$. This may be due to the fact that the magnitude of $F_{2}$ (and also $F_{1}$ ) varies highly, which is known as the 'dynamical range issue'. Here, resampling with the original function in rootsb leads to more accurate results at the expense of speed; see Figure 11. However, for larger $n$ as in Table 4, rootsb also does not compute the correct zeros. This highlights the ill-behavedness of the considered functions and how difficult it is to compute their zeros. The problem-adapted transport of images method finishes in all cases with the correct number of zeros; see Table 2. Moreover, it is much faster than Chebfun 2 and rootsb in these examples; see Figure 11 and Table 4.

\section{Summary and outlook}

We established and analyzed the transport of images method, which is the first problem-adapted method to compute all zeros of a (non-degenerate) 
harmonic mapping $f$. Our method is guaranteed to find all zeros of $f$, even though this number is a priori unknown, provided that no zero of $f$ is singular. Moreover, our MATLAB implementation performs remarkably well for several functions from the literature. While we focused on the computation of zeros in our examples, we can also compute all solutions of $f(z)=\eta$ for any point $\eta \in \mathbb{C} \backslash f(\mathcal{C})$. For this, we either consider $f-\eta$, or replace the transport path from $\eta_{1}$ to 0 by a transport path from $\eta_{1}$ to $\eta$ (with $\eta_{1}$ from the initial phase). More generally, we can compute all solutions of $f(z)=\eta$ given all solutions of $f(z)=\xi$ (with any points $\eta, \xi \in \mathbb{C} \backslash f(\mathcal{C})$ ) and the caustics of $f$, by transporting the solutions along a transport path from $\xi$ to $\eta$. This allows us to deduce the solutions for all right-hand sides from the local behavior of $f$ on the critical curves.

Further studies of the transport of images method could include different constructions of transport paths (e.g., step size control), improvements in the computation of the caustics, as well as the investigation of other algorithms as corrector. Generalizing the transport of images method to a broader class of functions, e.g., for harmonic mappings on domains with boundary (see, e.g., [4), might also be of interest. Finally, adjusting the transport of images method to the special functions of gravitational lensing should be of particular interest.

Acknowledgements We thank Jörg Liesen for several helpful suggestions on the manuscript. Moreover, we are grateful to the anonymous referees for valuable comments, which lead to improvements of this work.

\section{References}

[1] E. L. Allgower ANd K. GeOrg, Introduction to numerical continuation methods, vol. 45 of Classics in Applied Mathematics, Society for Industrial and Applied Mathematics (SIAM), Philadelphia, PA, 2003.

[2] J. Arango, H. Arbeláez, And J. Rivera, Orientation at singularities of harmonic functions, Monatsh. Math., 193 (2020), pp. 737-762.

[3] C. BÉnÉteau And N. Hudson, A survey on the maximal number of solutions of equations related to gravitational lensing, in Complex analysis and dynamical systems, Trends Math., Birkhäuser/Springer, Cham, 2018, pp. 23-38.

[4] W. Bergweiler And A. Eremenko, On the number of solutions of a transcendental equation arising in the theory of gravitational lensing, Comput. Methods Funct. Theory, 10 (2010), pp. 303-324.

[5] P. M. Bleher, Y. Homma, L. L. Ji, And R. K. W. Roeder, Counting zeros of harmonic rational functions and its application to gravitational lensing, Int. Math. Res. Not. IMRN, 2014 (2014), pp. 2245-2264.

[6] P. Duren, Harmonic mappings in the plane, vol. 156 of Cambridge Tracts in Mathematics, Cambridge University Press, Cambridge, 2004.

[7] P. Duren, W. Hengartner, and R. S. Laugesen, The argument principle for harmonic functions, Amer. Math. Monthly, 103 (1996), pp. 411-415. 
[8] C. D. Fassnacht, C. R. Keeton, and D. Khavinson, Gravitational lensing by elliptical galaxies, and the Schwarz function, in Analysis and mathematical physics, Trends Math., Birkhäuser, Basel, 2009, pp. 115-129.

[9] L. Geyer, Sharp bounds for the valence of certain harmonic polynomials, Proc. Amer. Math. Soc., 136 (2008), pp. 549-555.

[10] W. Hengartner and G. Schober, Univalent harmonic functions, Trans. Amer. Math. Soc., 299 (1987), pp. 1-31.

[11] P. Henrici, Applied and computational complex analysis. Vol. 3, Pure and Applied Mathematics (New York), John Wiley \& Sons, Inc., New York, 1986.

[12] K. L. JudD, Numerical methods in economics, MIT Press, Cambridge, MA, 1998.

[13] D. Khavinson And E. LundBerg, Transcendental harmonic mappings and gravitational lensing by isothermal galaxies, Complex Anal. Oper. Theory, 4 (2010), pp. 515524 .

[14] D. Khavinson and G. Neumann, On the number of zeros of certain rational harmonic functions, Proc. Amer. Math. Soc., 134 (2006), pp. 1077-1085.

[15] D. Khavinson and G. Neumann, From the fundamental theorem of algebra to astrophysics: a "harmonious" path, Notices Amer. Math. Soc., 55 (2008), pp. 666675 .

[16] D. Khavinson And G. Świastek, On the number of zeros of certain harmonic polynomials, Proc. Amer. Math. Soc., 131 (2003), pp. 409-414.

[17] S.-Y. Lee, A. Lerario, And E. Lundberg, Remarks on Wilmshurst's theorem, Indiana Univ. Math. J., 64 (2015), pp. 1153-1167.

[18] J. LIESEN AND J. ZUR, How constant shifts affect the zeros of certain rational harmonic functions, Comput. Methods Funct. Theory, 18 (2018), pp. 583-607.

[19] J. LiESEn AND J. ZuR, The maximum number of zeros of $r(z)-\bar{z}$ revisited, Comput. Methods Funct. Theory, 18 (2018), pp. 463-472.

[20] R. Luce And O. SÈTE, The index of singular zeros of harmonic mappings of antianalytic degree one, Complex Var. Elliptic Equ., 66 (2021), pp. 1-21.

[21] R. Luce, O. SÈte, And J. Liesen, Sharp parameter bounds for certain maximal point lenses, Gen. Relativity Gravitation, 46, Art. 1736 (2014), pp. 1-16.

[22] R. Luce, O. Sète, And J. Liesen, A note on the maximum number of zeros of $r(z)-\bar{z}$, Comput. Methods Funct. Theory, 15 (2015), pp. 439-448.

[23] A. Lyzzaik, Local properties of light harmonic mappings, Canad. J. Math., 44 (1992), pp. 135-153.

[24] S. Mao, A. O. Petters, And H. J. Witt, Properties of point mass lenses on a regular polygon and the problem of maximum number of images, in The Eighth Marcel Grossmann Meeting, Part A, B (Jerusalem, 1997), World Sci. Publ., River Edge, NJ, 1999, pp. 1494-1496.

[25] D. Mehta, T. Chen, J. D. Hauenstein, and D. J. Wales, Communication: Newton homotopies for sampling stationary points of potential energy landscapes, J. Chem. Phys., 141 (2014), p. 121104.

[26] A. Morgan, Solving polynomial systems using continuation for engineering and scientific problems, vol. 57 of Classics in Applied Mathematics, Society for Industrial and Applied Mathematics (SIAM), Philadelphia, PA, 2009.

[27] A. P. Morgan, A. J. Sommese, and C. W. Wampler, Computing singular solutions to nonlinear analytic systems, Numer. Math., 58 (1991), pp. 669-684. 
[28] A. P. Morgan, A. J. Sommese, and C. W. Wampler, A power series method for computing singular solutions to nonlinear analytic systems, Numer. Math., 63 (1992), pp. 391-409.

[29] Y. Nakatsukasa, V. Noferini, and A. Townsend, Computing the common zeros of two bivariate functions via Bézout resultants, Numer. Math., 129 (2015), pp. 181209.

[30] G. Neumann, Valence of complex-valued planar harmonic functions, Trans. Amer. Math. Soc., 357 (2005), pp. 3133-3167.

[31] J. M. ORtega And W. C. Rheinboldt, Iterative solution of nonlinear equations in several variables, Academic Press, New York-London, 1970.

[32] A. O. Petters, Gravity's action on light, Notices Amer. Math. Soc., 57 (2010), pp. 1392-1409.

[33] P. Schneider, J. Ehlers, and E. E. Falco, Gravitational Lenses, Springer Science \& Business Media, Berlin Heidelberg, 1999.

[34] O. SÈte, R. Luce, And J. Liesen, Creating images by adding masses to gravitational point lenses, Gen. Relativity Gravitation, 47, Art. 42 (2015), pp. 1-8.

[35] O. Sète, R. Luce, And J. Liesen, Perturbing rational harmonic functions by poles, Comput. Methods Funct. Theory, 15 (2015), pp. 9-35.

[36] O. SÈte And J. Zur, A Newton method for harmonic mappings in the plane, IMA J. Numer. Anal., 40 (2020), pp. 2777-2801.

[37] O. SÈTE AND J. ZUR, Number and location of pre-images under harmonic mappings in the plane, Ann. Acad. Sci. Fenn. Math., 46 (2021).

[38] T. Sheil-Small, Complex Polynomials, vol. 75 of Cambridge Studies in Advanced Mathematics, Cambridge University Press, Cambridge, 2002.

[39] S. Smale, Newton's method estimates from data at one point, in The merging of disciplines: new directions in pure, applied, and computational mathematics (Laramie, Wyo., 1985), Springer, New York, 1986, pp. 185-196.

[40] A. J. Sommese And C. W. Wampler, II, The numerical solution of systems of polynomials, World Scientific Publishing Co. Pte. Ltd., Hackensack, NJ, 2005.

[41] T. J. Suffridge And J. W. Thompson, Local behavior of harmonic mappings, Complex Variables Theory Appl., 41 (2000), pp. 63-80.

[42] A. Townsend and L. N. Trefethen, An extension of Chebfun to two dimensions, SIAM J. Sci. Comput., 35 (2013), pp. c495-c518.

[43] E. Wegert, Visual complex functions. An introduction with phase portraits, Birkhäuser/Springer Basel AG, Basel, 2012.

[44] A. S. Wilmshurst, The valence of harmonic polynomials, Proc. Amer. Math. Soc., 126 (1998), pp. 2077-2081. 